\title{
A Cdc42/RhoA regulatory circuit downstream of glycoprotein lb guides transendothelial platelet biogenesis
}

Sebastian Dütting ${ }^{1,2}$, Frederique Gaits-lacovoni ${ }^{3}$, David Stegner ${ }^{1}$, Michael Popp ${ }^{1,2}$, Adrien Antkowiak ${ }^{3}$, Judith M.M. van Eeuwijk1,2, Paquita Nurden 1,4, Simon Stritt1,2, Tobias Heib²,2, Katja Aurbach1,2, Oguzhan Angay², Deya Cherpokova 1,2, Niels Heinz ${ }^{5}$, Ayesha A. Baig1,2, Maximilian G. Gorelashvili,2, Frank Gerner 1,2, Katrin G. Heinze ${ }^{2}$, Jerry Ware ${ }^{6}$, Georg Krohne ${ }^{7}$, Zaverio M. Ruggeri ${ }^{8}$, Alan T. Nurden ${ }^{4}$, Harald Schulze ${ }^{1}$ Ute Modlich ${ }^{5}$, Irina Pleines ${ }^{1,2}$, Cord Brakebusch ${ }^{9} \&$ Bernhard Nieswandt $^{1,2}$

Blood platelets are produced by large bone marrow (BM) precursor cells, megakaryocytes (MKs), which extend cytoplasmic protrusions (proplatelets) into BM sinusoids. The molecular cues that control MK polarization towards sinusoids and limit transendothelial crossing to proplatelets remain unknown. Here, we show that the small GTPases Cdc42 and RhoA act as a regulatory circuit downstream of the MK-specific mechanoreceptor GPIb to coordinate polarized transendothelial platelet biogenesis. Functional deficiency of either GPIb or Cdc42 impairs transendothelial proplatelet formation. In the absence of RhoA, increased Cdc42 activity and MK hyperpolarization triggers GPIb-dependent transmigration of entire MKs into BM sinusoids. These findings position Cdc42 (go-signal) and RhoA (stop-signal) at the centre of a molecular checkpoint downstream of GPIb that controls transendothelial platelet biogenesis. Our results may open new avenues for the treatment of platelet production disorders and help to explain the thrombocytopenia in patients with Bernard-Soulier syndrome, a bleeding disorder caused by defects in GPIb-IX-V.

\footnotetext{
${ }^{1}$ Institute of Experimental Biomedicine, University Hospital and University of Würzburg, Josef-Schneider-Str. 2, 97080 Würzburg, Germany. ${ }^{2}$ Rudolf Virchow Center, University of Würzburg, Josef-Schneider-Str. 2, 97080 Würzburg, Germany. ${ }^{3}$ INSERM UMR1048, Institut des Maladies Métaboliques et Cardiovasculaires-I2MC, UMR1048, Institut National de la Santé et de la Recherche Médicale, Université de Toulouse, 1 Avenue Jean Poulhès, BP 84225, 31432 Toulouse Cedex 4, France. ${ }^{4}$ Institut Hospitalo-Universitaire LIRYC, Plateforme Technologique d'Innovation Biomédicale, Hôpital Xavier Arnozan, Avenue du Haut Lévêque, 33604 Pessac, France. ${ }^{5}$ Research Group for Gene Modification in Stem Cells, LOEWE Center for Cell and Gene Therapy Frankfurt/Main and the Paul-Ehrlich-Institute, Paul-Ehrlich-Straße 51-59, 63225 Langen, Germany. ${ }^{6}$ Department of Physiology and Biophysics, University of Arkansas for Medical Sciences, 4301 West Markham Street, Little Rock, Arkansass 72205, USA. 7 Biocenter, University of Würzburg, Am Hubland, 97074 Würzburg, Germany. ${ }^{8}$ Department of Molecular Medicine, The Scripps Research Institute, 10550 N Torrey Pines Rd, La Jolla, California 92037, USA. ${ }^{9}$ BRIC, Biomedical Institute, University of Copenhagen, Nørregade 10, 1165 Copenhagen, Denmark. Correspondence and requests for materials should be addressed to B.N. (email: bernhard.nieswandt@virchow.uni-wuerzburg.de).
} 
$\mathrm{B}$ lood platelets are anucleated cell fragments derived from bone marrow (BM) megakaryocytes (MKs) that are essential for blood clotting. MKs develop from haematopoietic stem cells by a complex maturation process, which includes DNA replication without cell division (endomitosis) and the formation of a demarcation membrane system (DMS), which functions as a membrane reservoir for newly formed platelets ${ }^{1,2}$. Mature BM MKs are giant, polyploid cells that localize close to $\mathrm{BM}$ sinusoids in order to extend and release long cytoplasmic protrusions called proplatelets into the sinusoidal lumen, from which platelets are shed under the influence of blood shear forces ${ }^{3-6}$. The highly controlled process of transendothelial platelet biogenesis stands in contrast to transendothelial migration of whole haematopoietic (progenitor) cells during mobilization and homing ${ }^{7}$. A current concept incorporates the idea that the chemokine stromal cell-derived factor-1 triggers migration of early MKs from the endosteal niche towards BM sinusoids ${ }^{8,9}$ and that the lipid mediator sphingosine-1-phosphate elicits directed proplatelet extension into the circulation ${ }^{10}$. However, the molecular mechanisms regulating the trafficking of MKs and haematopoietic cells within the BM and across the endothelial barrier are poorly defined.

Rho GTPases are small proteins $(20-25 \mathrm{kDa})$ belonging to the superfamily of Ras-related proteins which are found in all eukaryotic cells ${ }^{11}$. They are best known for regulating cytoskeletal dynamics in virtually all cell types ${ }^{12}$. The best-characterized Rho GTPases are Cdc42, RhoA and Racl, whose activation is associated with the formation of filopodia, stress fibres and lamellipodia, respectively. We previously reported that transgenic mice lacking either Cdc42 or RhoA in MKs and platelets exhibit pronounced macrothrombocytopenia, indicating a distinct role of these molecules in platelet production ${ }^{13,14}$. In addition, Cdc42 deficiency led to decreased filopodia formation of platelets on von Willebrand factor (vWF), suggesting a unique role of Cdc42 downstream of the glycoprotein (GP) Ib subunit of the vWF receptor complex GPIb-IX-V (ref. 13). Bernard-Soulier syndrome (BSS) is a rare platelet disorder characterized by macrothrombocytopenia, which is caused by damaging variants in either of the three genes encoding the GPIb $\alpha / \beta$ or GPIX subunits of the GPIb-IX-V complex, leading to its absence from, or dysfunction at the $M K$ and platelet surface ${ }^{15-19}$. The mechanisms by which the receptor controls megakaryopoiesis and platelet production are largely unknown.

Here, we show that lack of either functional GPIb or Cdc42 reduced MK polarization in vitro and impaired MK localization at sinusoids and transendothelial biogenesis in vivo. In contrast, absence of RhoA in MKs resulted in increased Cdc42 activity and GPIb-dependent transendothelial migration of entire MKs into BM sinusoids. These results reveal that $\mathrm{Cdc} 42$ and RhoA act as a regulatory circuit downstream of GPIb $\alpha$ to coordinate MK polarization and transendothelial platelet biogenesis in vivo.

\section{Results}

GPIb $\alpha$ signalling controls MK localization. Lack of functional GPIb-IX-V results in impaired DMS development and distribution during $\mathrm{MK}$ maturation in humans and mice $\mathrm{e}^{20,21}$. We analysed MK localization in the BM of mice lacking GPIb $\alpha$ $\left(G p 1 b a^{-/-}\right)$by immunostaining of native cryosections of whole femora (Fig. 1). In wild-type (wt) mice, the majority of MKs was in close contact to BM sinusoids $(61.5 \pm 4.7 \%$; Fig. 1a,b). Only rarely were MKs found within the sinusoidal lumen $(0.6 \pm 1.4 \%)$. Interestingly, in $G p 1 b a^{-/-}$mice the number of MKs with direct contact to sinusoids was significantly reduced (48.1 $\pm 4.0 \%$; $P=0.0002$; two-way ANOVA with Bonferroni correction for multiple comparisons) resulting in an increase in $\mathrm{BM}$ haematopoietic compartment (BMHC)-localized MKs (50.2 $\pm 6.3 \%$; $P=0.0005$; Fig. 1a,b).

In view of the multiple intrinsic defects present in MKs deficient in GPIb $\alpha^{22}$ or GPIb $\beta^{23}$ we turned to a mouse line expressing a mutant version of GPIb $\alpha$, where the ectodomain of GPIb $\alpha$ is replaced by that of the human interleukin- 4 receptor $\alpha$ $(\mathrm{IL}-4 \mathrm{R} \alpha)\left(G p 1 b a^{-/-; t g}\right.$, further referred to as $\left.G p 1 b a-T g\right)$. In these mice, the BSS-associated macrothrombocytopenia is ameliorated, but not fully reversed (Supplementary Fig. 1a,b (refs 21,24)), implying a specific role for the ectodomain of GPIb $\alpha$ in platelet biogenesis. Strikingly, the BM MK distribution in Gp1ba-Tg mice and Gp1ba-/- mice was similar, with a significant decrease in the proportion of MKs with sinusoidal contact ( $w t: 67.5 \pm 6.4 \% ; G p 1 b a-T g: 49.4 \% \pm 7.0 \% ; P<0.0001$ ) and a concomitant increase of BMHC-localized MKs (wt: $30.3 \pm 6.6 \% ; \quad G p 1 b a-T g: 49.0 \pm 6.0 \% ; P<0.0001$; two-way ANOVA with Bonferroni correction for multiple comparisons) (Fig. 2a,c). The role of the GPIb $\alpha$ ectodomain for MK localization was further confirmed by treatment of $w t$ mice with the monovalent Fab fragment of an antibody directed against the major ligand (vWF)-binding domain of GPIb $\alpha(\mathrm{p} 0 \mathrm{p} / \mathrm{B}-\mathrm{Fab})$ that is known not to affect platelet survival in the circulation ${ }^{25}$. While GPIb $\alpha$ blockade had no effect on total MK numbers in the BM (Supplementary Fig. 1c), it clearly reduced the fraction of MKs with direct sinusoidal contact $(39.5 \pm 4.4 \%$; $P<0.0001$; two-way ANOVA with Bonferroni correction for multiple comparisons, Fig. 2a,c). This was associated with a reduction in peripheral platelet counts and an increase in platelet size by about one third (Fig. 2d,e) as compared to $w t$, similar to that seen in Gp1ba-Tg mice (Supplementary Fig. 1a,b). Formation of the DMS, as analysed by transmission electron microscopy (TEM), was unaltered in MKs of Gplba-Tg mice or wt mice after GPIb $\alpha$ blockade (Fig. 2b). Together, these findings revealed a critical role for the ectodomain of GPIb $\alpha$ in controlling MK localization at vascular sinusoids, independent of its role in DMS development and partitioning.

We next sought to get insights into the signalling that triggers GPIb $\alpha$-dependent MK guidance within the BM. Using mice lacking $\mathrm{Cdc} 42$ in $\mathrm{MKs}$ and platelets $\left(C d c 42^{f l / f l}\right.$ Pft-cre, further referred to as $C d c 42^{-/-}$(ref. 13)), we have previously shown that Cdc42 controls cytoskeletal dynamics downstream of GPIb $\alpha$ in platelets and that its absence in MKs causes marked macrothrombocytopenia by unknown mechanisms ${ }^{13}$. Intriguingly, significantly fewer $C d c 42^{-/}$MKs were in direct contact with $\mathrm{BM}$ sinusoids in line with an increased $\mathrm{MK}$ population in the BMHC (Fig. 2f,g) compared to wt littermate controls $\quad(52.0 \pm 3.6 \quad P=0.0002$; two-way ANOVA with Bonferroni correction for multiple comparisons). Importantly, Cdc42 $2^{-/-}$MKs displayed only partially reduced membrane invaginations, indicating that the thrombocytopenia in these mice was not solely the result of a lack of intracellular membranes (Fig. 2h). Another key component of the GPIb $\alpha$ signalling machinery is phosphoinositide 3-kinase (PI3K) that has been shown to play an important role in thrombosis and GPIb $\alpha$ induced integrin activation ${ }^{26,27}$. Of note, treatment of $w t$ mice with the PI3K inhibitor wortmannin reduced the number of MKs in direct contact with sinusoids compared to the control (55.0 $\pm 5.9 \%, P=0.003$; two-way ANOVA with Bonferroni correction for multiple comparisons) (Supplementary Fig. 2d,e), whereas it had no effect on DMS development or total MK numbers in the BM (Supplementary Fig. 2a,b,f).

In neuronal cells, PI3K-mediated establishment of cell polarity was shown to involve the interplay of GTPases with atypical protein kinase $\mathrm{C}$ (aPKC) isoforms, which form part of the PAR ('partitioning defective') complex ${ }^{28}$. We observed reduced sinusoidal localization of MKs in mice lacking the aPKC isoform 
a

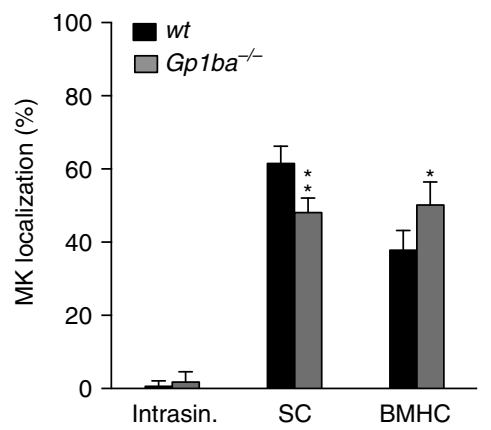

b

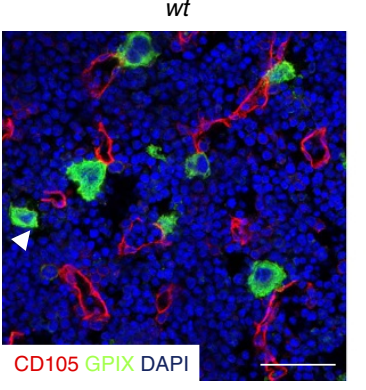

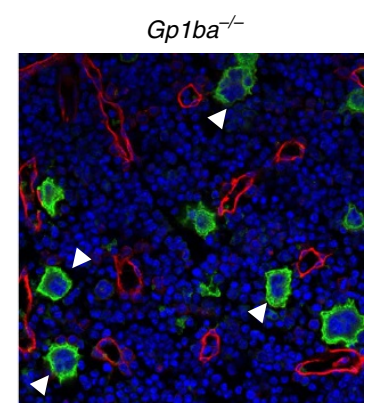

Figure 1 | GPIb $\boldsymbol{\alpha}$ deficiency alters MK localization in the BM. (a) Quantification of MK localization in the BM reveals less sinusoidal contact (SC) and increased localization in the BM haematopoietic compartment (BMHC) in Gp1ba-/- mice (grey) compared to the wild-type (wt, black) ( $n=5$ );

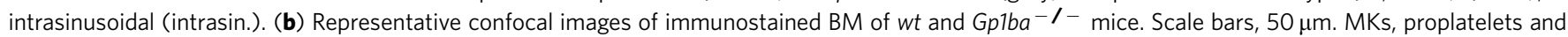
platelets are shown by CD41 staining in green. Endoglin staining (red) labels vessels. DAPI, blue. Arrowhead indicates MKs in the BM haematopoietic compartment $(B M H C)$. Bar graphs represent mean \pm s.d. Two-way ANOVA with Bonferroni correction for multiple comparisons; ${ }^{\star} P<0.01 ;{ }^{\star} P<0.05$.

$\mathrm{PKCl}$ in MKs/platelets (Supplementary Fig. 3a,b), indicating a role of the PAR complex and atypical PKCs for PI3K-Cdc42 controlled $\mathrm{MK}$ polarization and migration/localization. Regulation of cell polarization by the PAR complex is characterized by a profound reorganization of the microtubule cytoskeleton $^{29}$. Consistently, we found that microtubule disruption by nocodazole treatment prevented transmigration of RhoA-deficient MKs (Supplementary Fig. 3c,d, see below) similar to Cdc42-deficiency.

RhoA negatively regulates GPIb-Cdc42 signalling in MKs. Rho GTPases integrate phosphoinositide signalling and cytoskeletal dynamics. This involves a cross-regulation between Cdc42 and RhoA, which tunes GTPase function in different cell types $^{30,31}$. It was recently demonstrated that Cdc42 is important to polarize the MK DMS towards BM sinusoids ${ }^{32}$. Moreover, we have previously shown that mice with $\mathrm{MK} /$ platelet-specific RhoA-deficiency (RhoA ${ }^{f l f l}$ Pf4-cre, further referred to as $R h o A^{-/-}$) exhibit pronounced macrothrombocytopenia (Supplementary Fig. 4a,b). This finding was striking since it was reported that RhoA negatively regulates proplatelet formation of human $\mathrm{CD} 34+$ derived MKs in vitro ${ }^{33}$. Supporting this result, we found that proplatelet formation of $R h o A^{-}$- fetal liver cell-derived MKs in liquid culture in vitro was comparable to the $w t$ (Supplementary Fig. 4e (ref. 14)). Taking these observations together, we hypothesized that the apparent platelet biogenesis defect in $R h o A^{-/-}$mice might be mechanistically linked to defective control of GPIb $\alpha$-Cdc42 signalling and MK localization within the BM. Intriguingly, in BM sections of $R h o A^{-/-}$mice, we found a dramatic MK mislocalization with approximately $30 \%$ of the cells being present inside the BM sinusoids $(27.9 \pm 10 \%$, $P<0.0001$; two-way ANOVA with Bonferroni correction for multiple comparisons; Fig. 3a,b, Supplementary Fig. 4c,d). The remaining MKs were mostly found in direct contact with sinusoids $(61.2 \pm 8.6 \%, \quad P=0.430$; two-way ANOVA with Bonferroni correction for multiple comparisons), while only a very minor population was present in the BMHC $(10.8 \pm 2.3 \%$, $P<0.0001$; two-way ANOVA with Bonferroni correction for multiple comparisons). To analyse the ultrastructure of intrasinusoidal MKs in the intact BM, we performed TEM on cross-sections of intact femora (Fig. 3c, Supplementary Fig. 5). Intrasinusoidal $R h o A^{-/-}$MKs showed signs of maturation including poly-lobulated nuclei. The MKs either contained a large quantity of cytoplasm with a well-developed membrane system and a pronounced peripheral zone, or showed signs of flowdependent DMS/cytoplasm detachment suggestive of proplatelet formation. Nevertheless, most MKs remained in contact with sinusoidal endothelial cells. Of note, DMS formation appeared mostly unaffected in $R h o A^{-/-}$MKs when analysed by TEM on BM cross-sections (Supplementary Fig. 5c), but we frequently found intact cells of other lineages (typically granulocytes) within the MKs cytoplasm (Fig. 3c, Supplementary Fig. 5d), a phenomenon known as emperipolesis 34 .

Next, we utilized intravital two-photon microscopy (2PM) for dynamic visualization of $\mathrm{MK}$ localization and proplatelet formation in the BM of the skull over time. As previously described $^{4}$, in $w t$ mice multiple MKs were in direct sinusoidal contact and eventually released long proplatelets into the vascular sinus (Fig. 3d-f, Supplementary Movie 1). In sharp contrast, in $R h o A^{-1-}$ mice, intravital proplatelet formation was increased (Supplementary Fig. 6a) and entire MKs (i) transmigrated from the BM compartment into the vascular sinus (Fig. 3e, Supplementary Movie 2, Supplementary Fig. 6b), (ii) adhered for up to $30 \mathrm{~min}$ (maximal observation period) inside the vascular lumen (Fig. 3f, Supplementary Movies 3 and 4) or (iii) extended and eventually released very large proplatelet-like fragments into the circulation (Supplementary Fig. 6c, Supplementary Movie 5). Although the pulmonary microvessels represent the first capillary bed that receives circulating blood from the $\mathrm{BM}^{35}$, we did not observe increased numbers of MKs in the lungs of Rho $A^{-1-}$ mice (Supplementary Fig. 6d,e), suggesting that the intrasinusoidal MKs predominantly fragment in the BM vasculature or elsewhere in the circulation ${ }^{3}$. These observations collectively indicate that RhoA negatively regulates MK guidance/ localization towards BM vascular sinusoids and thereby acts as a key regulator of proplatelet extension across the endothelial barrier by providing a stop-signal for MK transmigration.

To assess whether the transmigration of RhoA ${ }^{-/-}$MKs was indeed GPIb $\alpha$-dependent, we blocked the major ligand-binding site of the receptor in $w t$ and $R h o A^{-/-}$mice by injection of $\mathrm{p} 0 \mathrm{p} /$ $\mathrm{B}-\mathrm{Fab}$. Strikingly, whereas this treatment did not affect overall MK numbers in the BM (Supplementary Fig. 7), it reduced the intrasinusoidal localization of $R h o A^{-/}$MKs by more than $70 \%(8.2 \pm 4.4 \%, P<0.001$; two-way ANOVA with Bonferroni correction for multiple comparisons, Fig. 4a,d). This was associated with an increased presence of MKs in direct contact with BM sinusoids and a decreased percentage of BMHClocalized MKs compared with wt controls and vehicle-treated Rho $A^{-/-}$mice, respectively (Fig. 4d). In contrast, treatment with Fab fragments of antibodies directed against the major platelet integrin $\alpha \mathrm{IIb} \beta 3$ (JON/A (ref. 36)) or GPV (DOM1 (ref. 37)) did neither affect $\mathrm{MK}$ localization in the $w t$ 
(Supplementary Fig. 7b,c), nor was it able to revert the transendothelial migration of RhoA $A^{-/-}$MKs, demonstrating a specific role for GPIb in these processes. We also generated
RhoA $A^{-/-}$Gplba-Tg mice, in which we found similar results as compared to $\mathrm{p} 0 \mathrm{p} / \mathrm{B}-\mathrm{Fab}$ treatment $(9.5 \pm 4.5 \% \mathrm{MKs}$ intrasinusoidal, Fig. 4b,d). Interestingly, inhibition of PI3K by

a
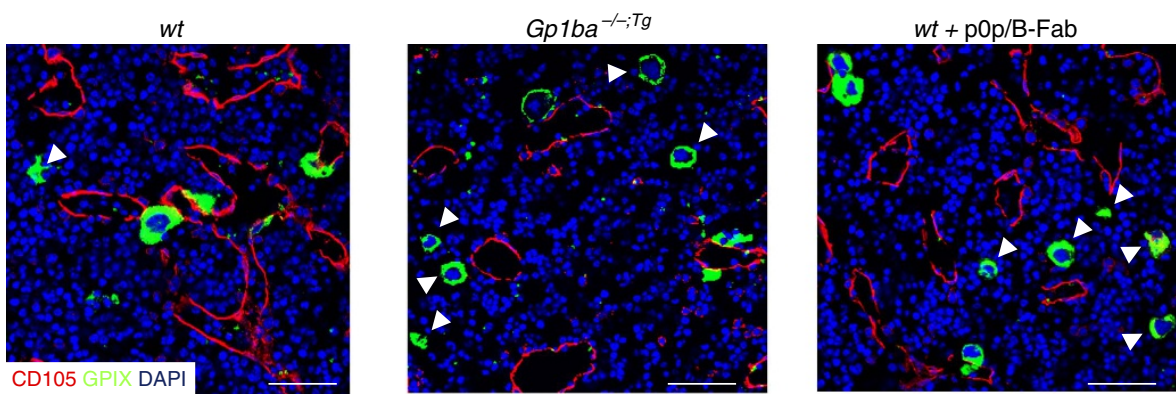

b
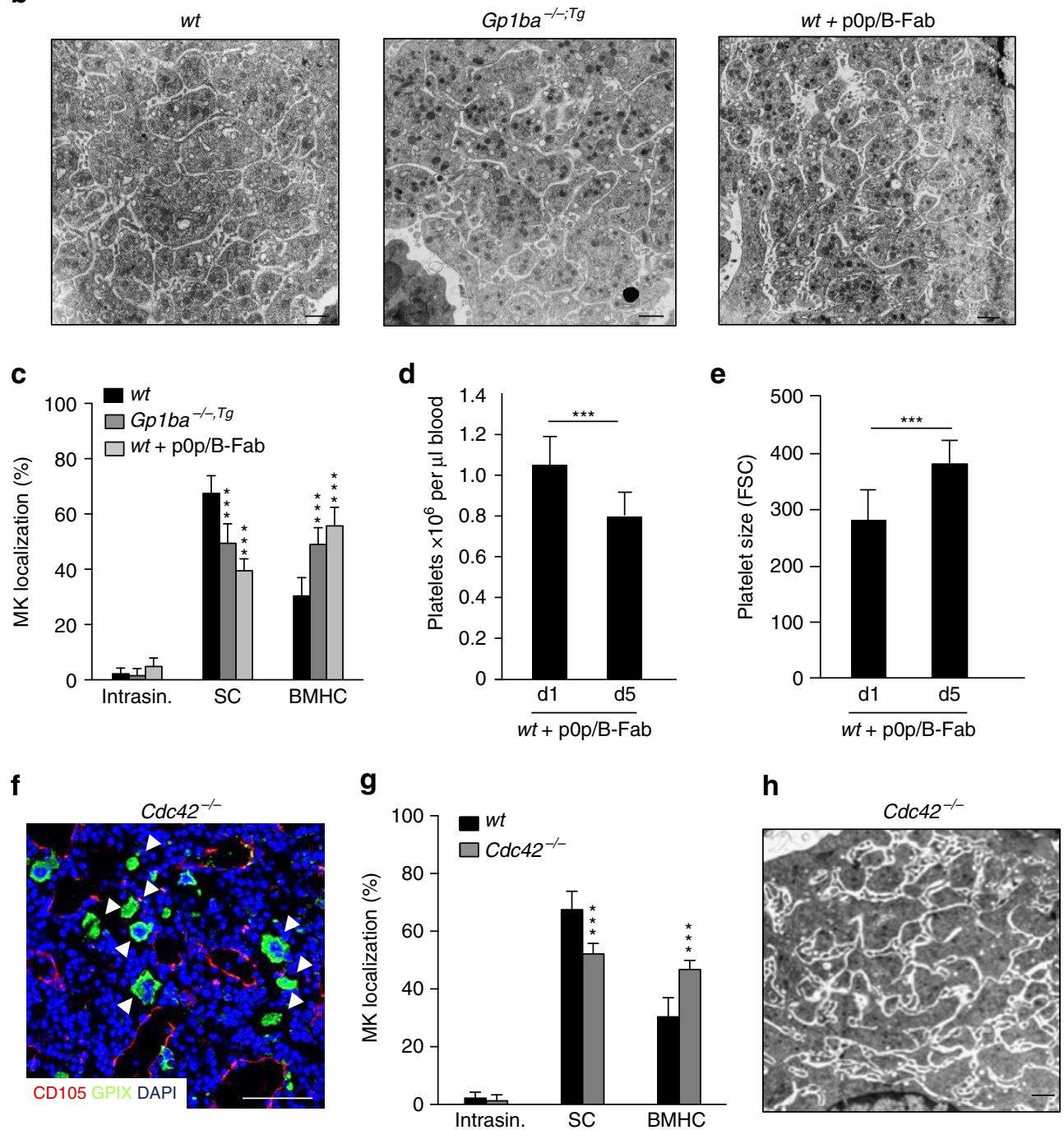

Figure 2 | GPIb $\alpha$ ectodomain and Cdc42 regulate MK localization in the BM. (a) Confocal images of immunostained BM and (b) TEM analysis of BM MKs of wt (left panel), Gplba-/-;tg (Gplba-Tg) (middle panel) and wt mice after treatment with GPIb $\alpha$-blocking monovalent Fab fragments, pOp/B-Fab (right panel). $n=4$ biological replicates. Scale bars, $50 \mu \mathrm{m}$ (a) and $2 \mu \mathrm{m}$ (b). MKs, proplatelets and platelets are shown by GPIX staining in green. Endoglin staining (red) labels vessels. DAPI, blue. Arrowhead indicates MKs in the BM haematopoietic compartment (BMHC). (c) Quantification of MK localization in the BM reveals less sinusoidal contact (SC) in Gp1ba-Tg (dark grey) and wt mice after GPIb $\alpha$-blockade (light grey) compared to the wt (black) $(n=7,5$ and 10); intrasinusoidal (intrasin.). (d,e) Reduced platelet count (d) and increased platelet size (e) in wt mice after GPIb $\alpha$-blockade $(n=4)$. (f) Representative confocal images of immunostained BM of $\mathrm{Cdc}_{2} 2^{-/-}$mice. Scale bar, $50 \mu \mathrm{m}$. (g) Quantification of MK localization in the BM reveals reduced SC in wt (black) and Cdc42 $2^{-1-}$ (grey) mice $\left(n=10\right.$ and 3). (h) TEM analysis of BM MKs of $C d c 42^{-/-}$mice. Scale bar, $2 \mu \mathrm{m}$. Bar graphs represent mean \pm s.d. $(\mathbf{c}, \mathbf{g})$ Two-way ANOVA with Bonferroni correction for multiple comparisons; $(\mathbf{d}, \mathbf{e})$ Unpaired two-tailed Student's $t$-test; ${ }^{* \star \star} P<0.001$. 
a
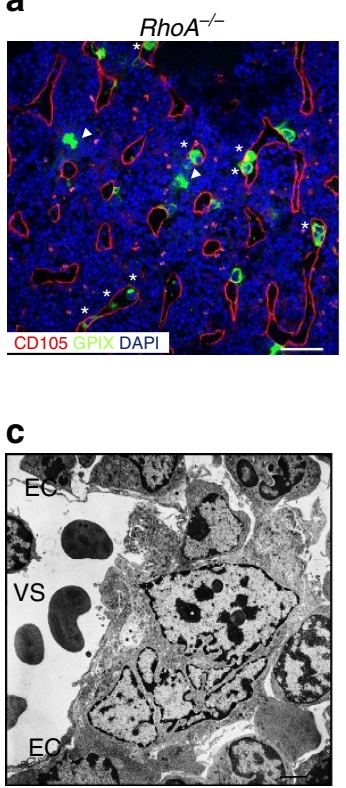

d
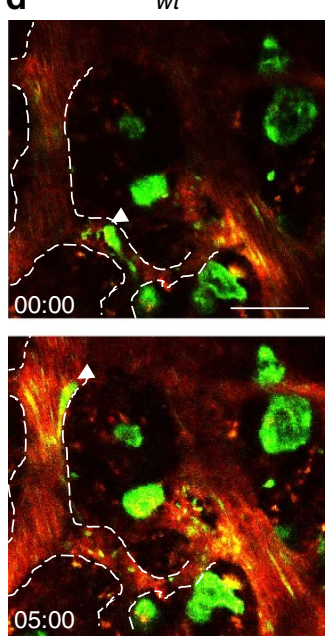

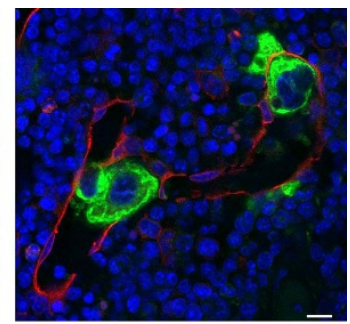

b
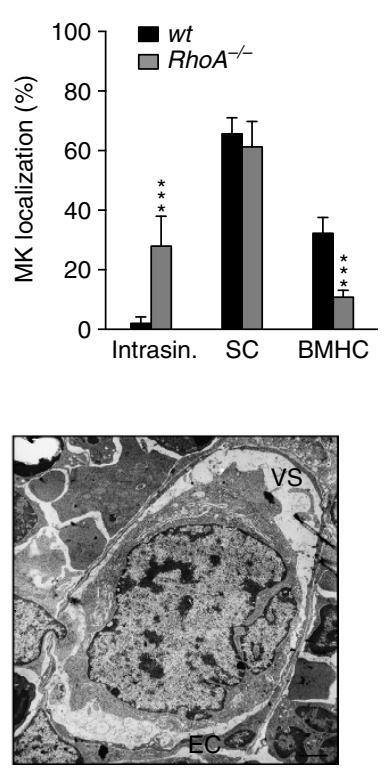

e
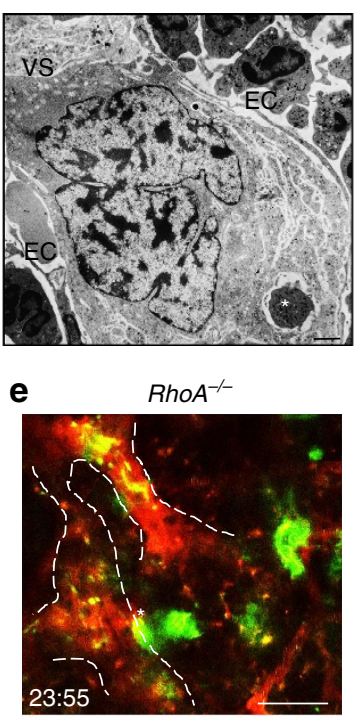

f
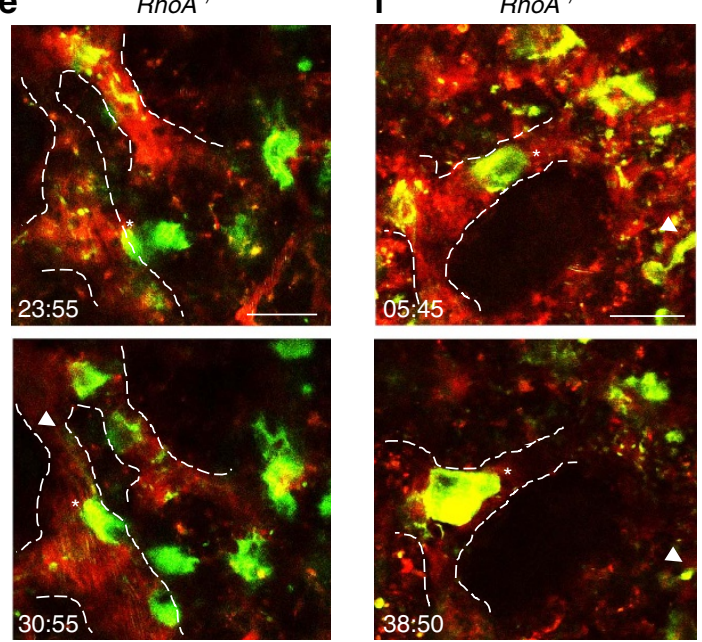

Figure 3 | RhoA negatively regulates MK guidance and transmigration. (a) Representative confocal images of immunostained $B M$ of $R h o A^{-/-}$mice. Scale bars, $50 \mu \mathrm{m}$ (left panel); $10 \mu \mathrm{m}$ (right panel). MKs, proplatelets and platelets are shown by GPIX staining in green. Endoglin staining (red) labels vessels. DAPI, blue. Arrowhead indicates MKs in BMHC, asterisk indicates intrasinusoidal (intrasin.) MKs. (b) Quantification of MK localization in the BM reveals abundant intrasinusoidal localization of $R h A^{-/-}$(grey) MKs compared to the wt (black) $(n=9)$. (c) TEM analysis of RhoA ${ }^{-/-}$BM MKs. Scale bars, $2.5 \mu \mathrm{m}$. EC, endothelial cell; VS, vascular sinus. Asterisk indicates emperipolesis $\left(n=6\right.$ (wt) and 8 (Rho $\left.A^{-/-}\right)$). (d-f) Intravital two-photon microscopy of wt (d) and $R h o A^{-/-}(\mathbf{e}, \mathbf{f})$ BM MKs in the skull. Arrowhead indicates proplatelet formation, asterisk indicates intrasinusoidal MKs $\left(n=7\right.$ and 6). Scale bars, $50 \mu \mathrm{m}$. Bar graphs represent mean \pm s.d. Two-way ANOVA with Bonferroni correction for multiple comparisons; ${ }^{\star \star \star} P<0.001$.

wortmannin treatment likewise significantly reduced the number of intrasinusoidal $\mathrm{MKs}$ in $R h o A^{-/-}$mice $(12.3 \pm 5.2 \%$, $P<0.0001$; two-way ANOVA with Bonferroni correction for multiple comparisons), but did not increase the number of MKs with direct sinusoidal contact (Supplementary Fig. 2d,e). Total MK numbers in the BM were unaltered in wortmannin-treated compared to vehicle-treated $R h o A^{-/-}$mice (Supplementary Fig. 2f) and also their DMS appeared largely unaltered (Supplementary Fig. 2c).

Together, these data indicate that RhoA may act as a negative regulator of GPIb $\alpha$-Cdc42-driven $\mathrm{MK}$ guidance towards $\mathrm{BM}$ sinusoids and transendothelial migration. To further test this hypothesis, we generated $\mathrm{MK} /$ platelet-specific RhoA/Cdc42double-deficient mice (RhoA/Cdc42flfl Pft-cre, further referred to as RhoA/Cdc42-/-; Supplementary Figs $8 \mathrm{a}$ and $11 \mathrm{a}-\mathrm{c}$ ). Strikingly, RhoA/Cdc42-I- MKs massively accumulated around vascular sinusoids $(82.9 \pm 5.9 \%, P=0.0002$; two-way ANOVA with Bonferroni correction for multiple comparisons, Fig. $4 \mathrm{c}-\mathrm{e}$, Supplementary Movie 6), but failed to transmigrate into the lumen $(3.9 \pm 1.8 \%$ MKs intrasinusoidal, Fig. $4 \mathrm{c}, \mathrm{e})$ or to efficiently form transendothelial proplatelets in vivo (Fig. 4f,g, Supplementary Movie 6). Consequently, these animals were severely thrombocytopenic (Supplementary Fig. 8b,c). Of note, intrasinusoidal localization of $R h o A^{-/-}$MKs was not altered by the concomitant lack of Racl (RhoA/Racl ${ }^{-/}$) (Supplementary Fig. 9a-e), thus indicating a specific RhoA-Cdc42 crosstalk downstream of GPIb $\alpha$ in this process.

RhoA controls MK polarization by limiting Cdc42 activity. We next investigated whether a possible crosstalk between Cdc42 and RhoA regulates MK polarization. Importantly, mature 
a

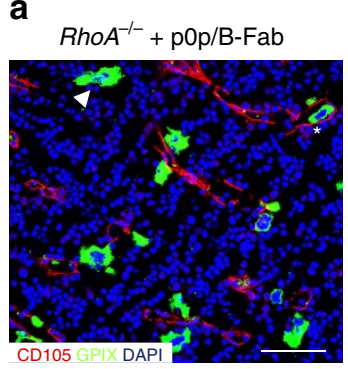

b

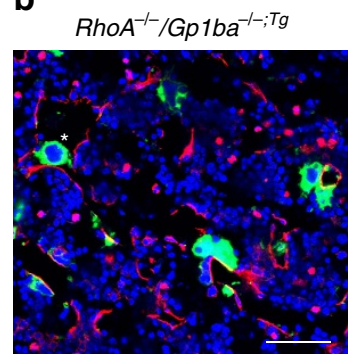

C

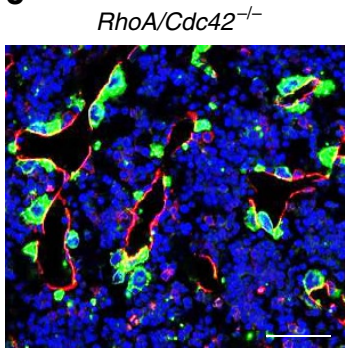

d

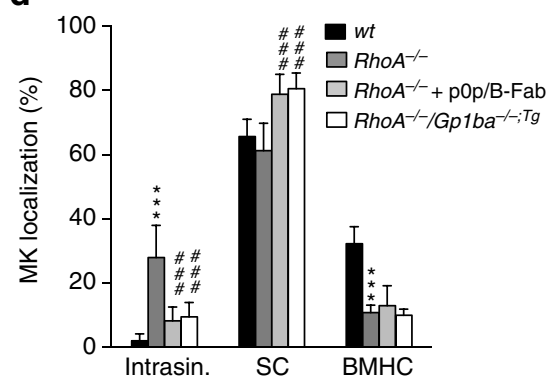

e

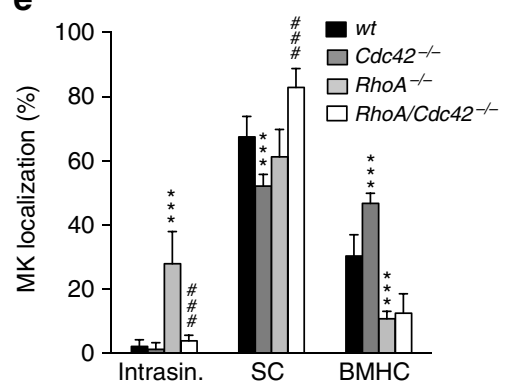

f

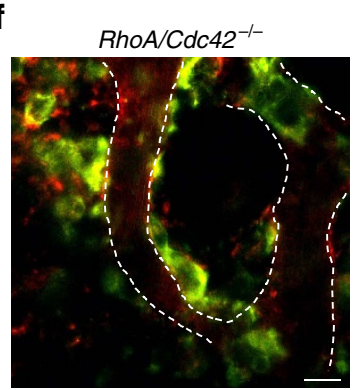

$\mathbf{g}$

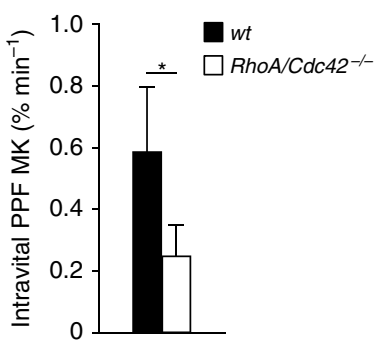

Figure 4 | RhoA restricts GPIb $\alpha$-Cdc42-driven MK localization and transendothelial migration. (a-c) Representative confocal images of immunostained $\mathrm{BM}$ of RhoA ${ }^{-/-}$mice after treatment with GPIb $\alpha$-blocking monovalent Fab fragments, pOp/B-Fab (a), concomitant lack of the GPIb $\alpha$ ectodomain (b), or RhoA/Cdc42 ${ }^{-/-}$mice (c). $n=4$ biological replicates. Scale bars, $50 \mu \mathrm{m}$. MKs, proplatelets and platelets are shown by GPIX staining in green colour. Endoglin staining (red) labels vessels. DAPI, blue. Arrowhead indicates MKs in BMHC, asterisk indicates intrasinusoidal (intrasin.) MKs. (d) Quantification of MK localization in the BM of RhoA ${ }^{-/-}$mice reveals reduced intrasinusoidal localization upon concomitant blockade (light grey) or absence (white) of GPIb $\alpha$ compared to normal $R h o A^{-/-}$mice (dark grey) ( $n=9,3$ and 9 ). Untreated wt is shown in black ( $n=9$ biological replicates). (e) Quantification of MK localization in the BM of RhoA/Cdc42-/- mice reveals reduced intrasinusoidal localization compared to RhoA ${ }^{-/-}$mice (light grey) and MK clustering at sinusoids ( $n=4$ and 9). Wt (black): $n=10, C d c 42^{-/-}$mice (dark grey); $n=3$. (f) Intravital two-photon microscopy of wt (black) and RhoA/Cdc42-/- MKs (white) in the skull ( $n=7$ and 4). Scale bar, $50 \mu \mathrm{m}$. (g) Quantification reveals reduced proplatelet-formation (ppf). Bar graphs represent mean \pm s.d. (d,e) Two-way ANOVA with Bonferroni correction for multiple comparisons; (g) Unpaired two-tailed Student's $t$-test; ${ }^{\star} P<0.05$; ${ }^{\star \star \star} P<0.001$ compared to $w t ;{ }^{*} \# P<0.001$ compared to $R$ hoA ${ }^{-/-}$.

$C d c 42^{-/-}$and Gp1ba-Tg BM MKs displayed defective DMS polarization after 4 days of liquid culture in vitro (Fig. $5 \mathrm{a}-\mathrm{c}, \mathrm{f}$ ), whereas increased polarization of not fully mature DMS was observed in RhoA $A^{-/-}$MKs (Fig. 5d,g). Strikingly, in vitro treatment with p0p/B-Fab (anti-GPIb) markedly reduced DMS polarization in cultures of both $w t$ and $R h o A^{-/-}$BM MKs (Fig. 5e,g), suggesting that the regulatory role of GPIb $\alpha$ in this process depends on intracytoplasmic GPIb-IX-V signalling and may operate in the absence of an ectopic ligand.

To decipher the underlying mechanism, we took advantage of lentiviral FRET biosensors derived from the Raichu probe to monitor Cdc42 activity in the polarized $\mathrm{DMS}^{32}$. Wt MKs showed a polarized Cdc42 activity at the DMS/F-actin complex, which was reduced by $68 \%$ in Gp1ba-Tg MKs (Fig. 6a,b,d), demonstrating the importance of controlled Cdc42 activation by GPIb $\alpha$ for correct DMS polarization. Strikingly, polarized Cdc42 activity showed a 2.4-fold increase in the absence of RhoA
(Fig. 6c,d), indicating that RhoA controls DMS and subsequently MK polarization by limiting Cdc42 activity. In order to determine how the nucleotide state of $\mathrm{Cdc} 42$ and RhoA influences this regulation, we utilized single amino acid mutants Cdc42/F28L and RhoA/F30L known to induce a constitutive exchange of GDP for GTP, thus resulting in hyperactive GTPases ${ }^{38}$. To restrict the expression of these mutants to the megakaryocytic lineage, we generated BM chimeric mice by transplantation of HSCs after lentiviral gene transfer, in which proteins were expressed under transcriptional control of the MK/platelet-specific human GP6 promoter (Fig. 7a,b) ${ }^{39}$. Of note, control mice transplanted with GFP-expressing HSCs showed reduced sinusoidal MK localization compared with non-irradiated mice $(44.3 \pm 2.94 \%$, Fig. 7c,f), indicating that irradiation influences MK localization. Compared to GFP-positive control cells, vessel association of Cdc42/F28L expressing MKs $\left(\mathrm{GFP}^{+} / \mathrm{GPIX}^{+}\right)$was increased $(57.0 \pm 9.0 \%$, Fig. $7 c, d, f)$ and approx. $4 \%$ of mutant MKs were 
a

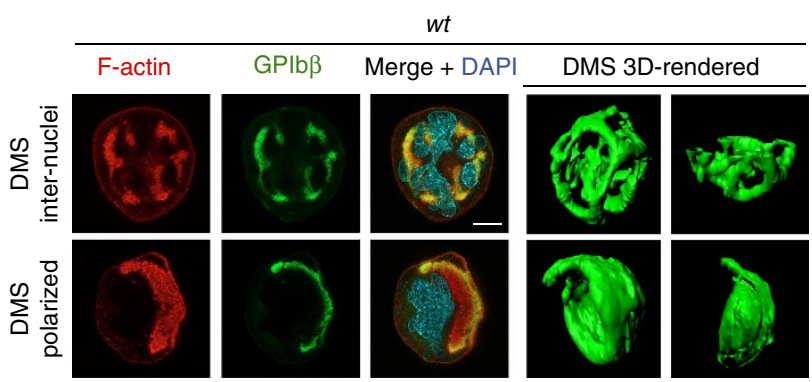

b

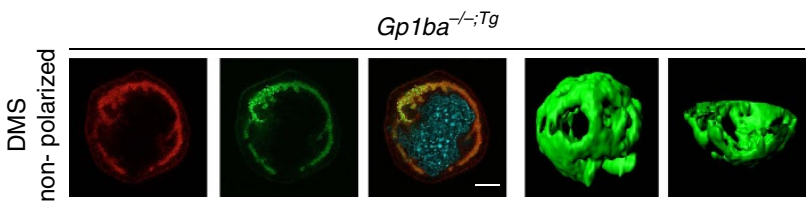

C

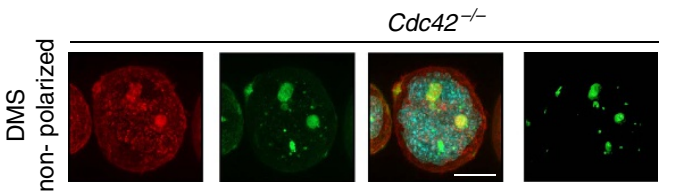

d

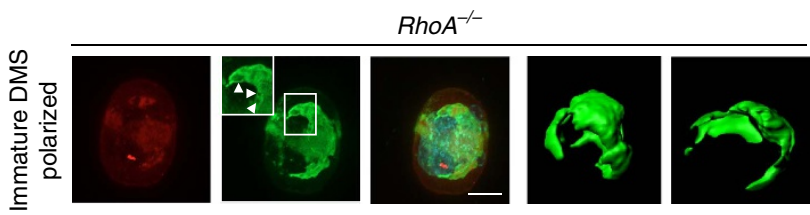

e

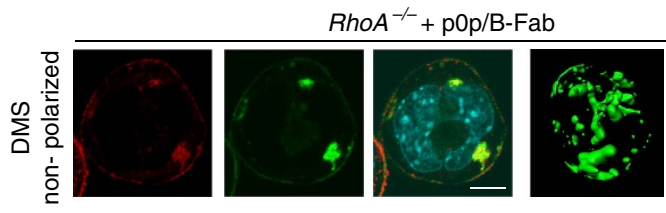

f

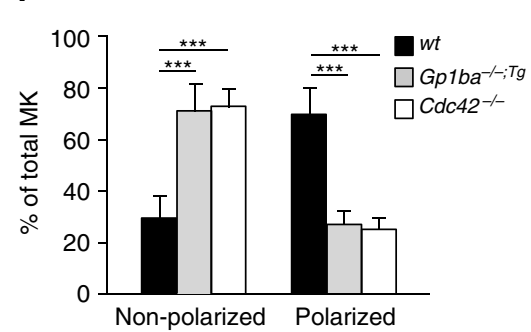

g

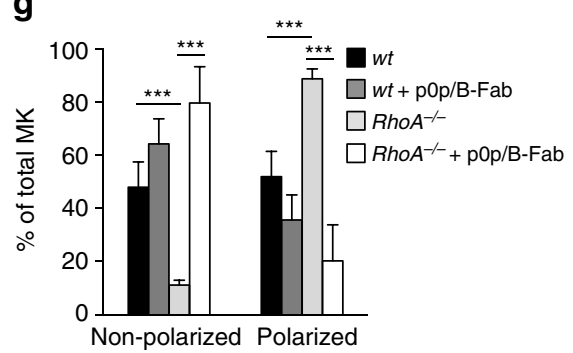

Figure 5 | RhoA controls GPIb $\boldsymbol{\alpha} / \mathbf{C d c 4 2 - d e p e n d e n t ~ M K ~ p o l a r i z a t i o n . ~ ( a - e ) ~ R e p r e s e n t a t i v e ~ c o n f o c a l ~ i m a g e s ~ o f ~ i n ~ v i t r o ~ d i f f e r e n t i a t e d ~ M K s ~ r e v e a l ~ p o l a r i z e d ~}$ DMS in mature wt MKs (a), defective polarization in Gplba-/-;Tg (Gplba-Tg) (b) and Cdc42-/- MKs (c), increased polarization in RhoA ${ }^{-/-}$MKs (d) and defective polarization of RhoA ${ }^{-/-}$MKs after treatment with GPIb $\alpha$-blocking monovalent Fab fragments, pOp/B-Fab (e). F-actin is stained by phalloidin (red); GPIb $\beta$ (a-d) or GPIX (e), green; DAPI, blue. 3D surface rendering of respective z-stacks (Imaris software; right panel). $n=5$ biological replicates. Scale bar: $10 \mu \mathrm{m}$. (f) Quantification of DMS polarization shows reduced MK polarization in Gp1ba- $\mathrm{Tg}$ (light grey) and Cdc42 $2^{-/-}$(white) MKs compared to the wt (black) ( $n=5$ biological replicates). (g) MK polarization is increased in RhoA $-/-$ MKs (light grey) compared to the untreated wt (black) and wt MKs treated with p0p/B-Fab fragments (dark grey). Treatment of RhoA $-/$ - MKs with p0p/B-Fab fragments (white) reverts the hyperpolarization. $n=5$ biological replicates ( $w t$ and $R h o A^{-/-}$) and 2 biological replicates ( $w t+\mathrm{pOp} / \mathrm{B}-\mathrm{Fab}$ and $R h o A^{-/-}+\mathrm{pOp} / \mathrm{B}-\mathrm{Fab}$ ). Bar graphs represent mean \pm s.d. Two-way ANOVA with Bonferroni correction for multiple comparisons; ${ }^{\star \star \star} P<0.001$.

located inside the BM sinusoids, whereas none were found in this compartment in control mice (Fig. 7c,d,f). In contrast, RhoA/F30L expression resulted in a further reduction of MKs in direct contact with BM sinusoids $(30.4 \pm 6.4 \%$, Fig. 7c,e,f) confirming the opposing functions of active Cdc42 (go-signal) and RhoA (stop-signal) in controlling MK localization and transendothelial proplatelet formation (Fig. 8).

\section{Discussion}

MKs are unique among haematopoietic progenitor cells in that they normally do not cross the endothelial lining of BM sinusoids, but extend and release portions of their cytoplasm into the blood stream by so far unknown mechanisms ${ }^{4,5}$. We now provide compelling evidence that this key step of unidirectional transendothelial proplatelet formation is tightly controlled 
a
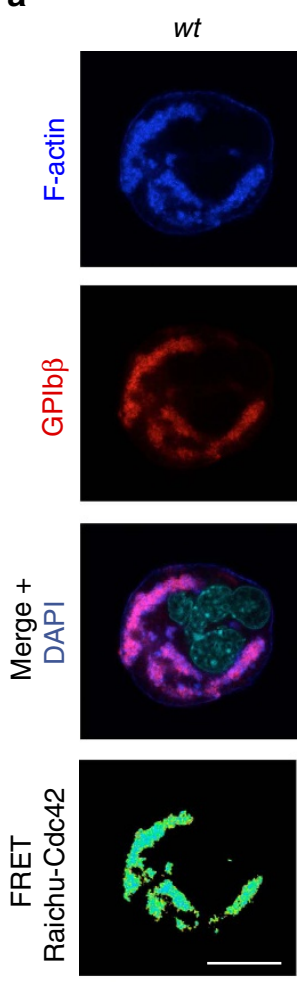

$1.1 \quad 7.0$

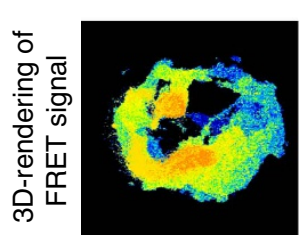

b
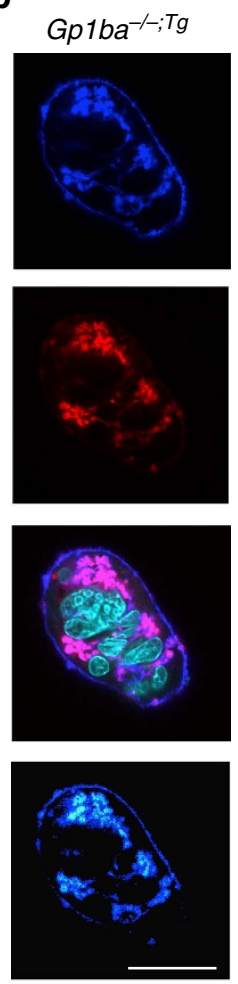

$1.0 \quad 5.6$

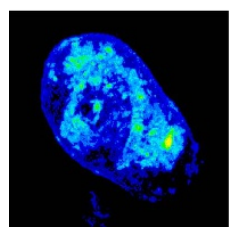

d

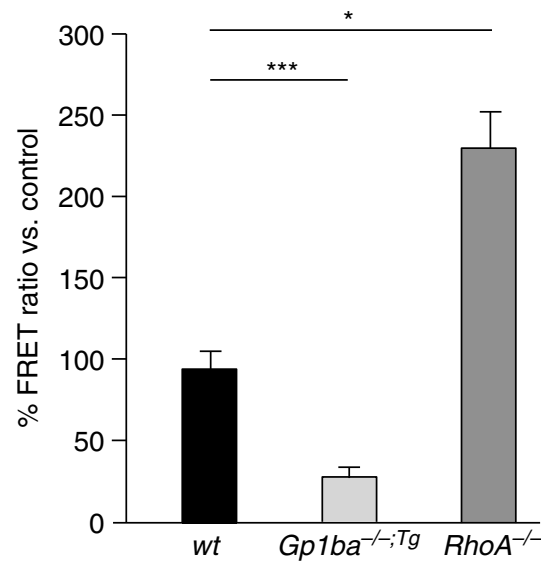

Figure 6 | RhoA controls GPIb $\alpha$-induced MK polarization by limiting

Cdc42 activity. (a-c) Cultured mouse MKs were transduced with Raichu-Cdc42 lentiviral vector. F-actin was stained with phalloidin (blue); GPIb $\beta$, red; DAPI, cyan. Cdc42 activation was visualized by colour-coded FRET. High ratio values (red) correlate with higher Cdc42 activity. Active Cdc42 localizes with the polarized F-actin/DMS complex and its activity is decreased in Gplba $a^{-/-i T g}$ (Gplba-Tg) and increased in RhoA ${ }^{-/-}$MKs. Scale bar: $10 \mu \mathrm{m}$. (d) Quantification reveals decreased Cdc42 activity in $\mathrm{Gp}_{\mathrm{b}} \mathrm{a}^{-/-; \mathrm{Tg}}$ (light grey) and increased activity in $\mathrm{Rho} \mathrm{A}^{-/-}$(dark grey) polarized MKs compared to the wt (black) ( $n=4$ biological replicates). Bar graphs represent mean \pm s.d. Two-way ANOVA with Bonferroni correction for multiple comparisons; ${ }^{\star} P<0.05$; ${ }^{\star \star \star} P<0.001$. within MKs by a crosstalk of $\mathrm{Cdc} 42$ and RhoA and requires a functional GPIb $\alpha$ ectodomain to occur efficiently (Fig. 8). Our data reveal a central antagonistic role of Cdc42 and RhoA in MK polarization and suggest that absence of RhoA supports locally increased Cdc42 activity and thereby directs the sites of cell protrusion. Our findings also highlight a critical function of the GPIb $\alpha$ ectodomain for efficient MK polarization, Cdc42 activation and thus sinusoidal localization and transendothelial proplatelet formation of mature MKs. Thus, these data indicate that MK mislocalization within the BM contributes to the low platelet counts in BSS patients and may mechanistically explain the defective transendothelial (pro-) platelet formation by impaired DMS polarization and Cdc42 activation.

Of note, vWF-deficient humans and mice display normal platelet counts ${ }^{40}$, and $\mathrm{MK}$ localization in the BM was not altered in $V w f^{-1-}$ mice (Supplementary Fig. 10a,b), which excludes a major role of this ligand in GPIb $\alpha$-dependent MK polarization.

Besides the vWF binding site, the $\mathrm{p} 0 \mathrm{p} / \mathrm{B}$ antibody Fab fragment also completely blocks thrombin binding to mouse GPIb $\alpha$ (Supplementary Fig. 10c), raising the possibility that this process might play a role in MK localization/polarization. However, MK localization in the BM of mice expressing human GPIb $\alpha$ with a mutation $(\mathrm{D} 277 \mathrm{~N})$ that abolishes $\alpha$-thrombin binding ${ }^{41}$ was similar to that of mice expressing $w t$ hGPIb $\alpha$ (Supplementary Fig. 10d,e), arguing against a role of thrombin in this process. Intriguingly, however, in vitro treatment of cultured MKs with p0p/B-Fab was sufficient to almost completely revert the DMS hyperpolarization observed in RhoA-deficient MKs. Thus, our findings do not indicate that GPIb $\alpha$ requires binding of an ectopic ligand to control MK localization and polarization in the BM. Rather, our results support the hypothesis that GPIb $\alpha$-mediated regulation of MK polarization might be a cell intrinsic process, which can be modulated by altering GPIb $\alpha$ signalling through changing the conformation and/or membrane localization/ clustering of the receptor in response to $\mathrm{p} 0 \mathrm{p} / \mathrm{B}$-Fab binding. In line with this, enhanced endogenous binding of mutant vWF to GPIb $\alpha$ in patients with vWF disease-type $2 \mathrm{~B}$ results in giant platelets and thrombocytopenia and was reported to be associated with dysregulation of the LIM kinase/cofilin pathway in MKs, together with upregulated RhoA signalling ${ }^{42}$. Thus, altered GPIb-IX-V-mediated intracellular signalling can lead to abnormal cytoskeletal signalling and severely abnormal MK function.

Taken all observations together, our data support a model in which GPIb $\alpha$ controls MK localization and transendothelial MK migration through intracellular signals, namely through the regulation of cell polarization via the Rho GTPases RhoA and Cdc42. While this process presumably involves MK PI3K function, we cannot rule out that treatment with the PI3K inhibitor wortmannin also exerts effects on vascular cells. Thus, deciphering the exact role of PI3K and/or PIs in MK localization requires further investigation. On the other hand, it is well known that $\mathrm{Cdc42}$ and PI3K play a central role in establishing morphological and molecular cell polarity in eukaryotic cells ${ }^{28,29}$. In neurons, establishment of cell polarity during axon specification requires PI3K-dependent crosstalk of GTPases with aPKC isoforms which form part of the PAR complex ${ }^{28}$. Consistently, Cdc42-mediated cell polarity in migrating astrocytes occurs through activation of the aPKC isoform $\mathrm{PKC}^{28,43}$. Thus, our observation of reduced sinusoidal localization of MKs in wortmannin-treated mice and mice deficient in the aPKC- isoform $\mathrm{PKC} 1$ is supportive of a role of PI3K signalling for Cdc42/RhoA-controlled MK polarization and localization.

During the last years, experimental evidence has accumulated suggesting that MKs possess an intrinsic network involving the 
a

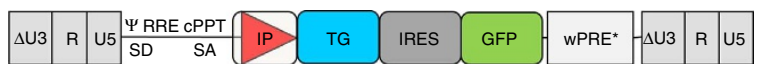

b
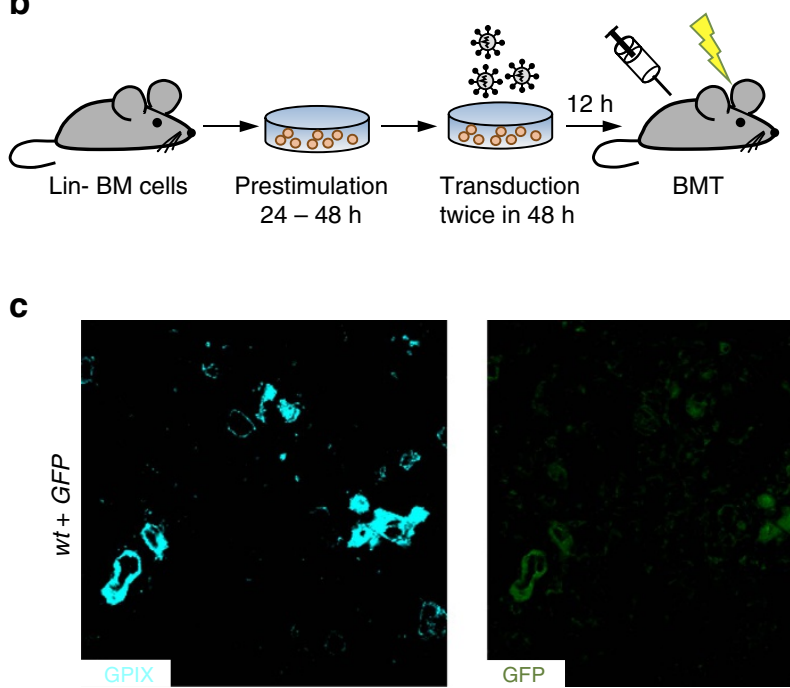

d
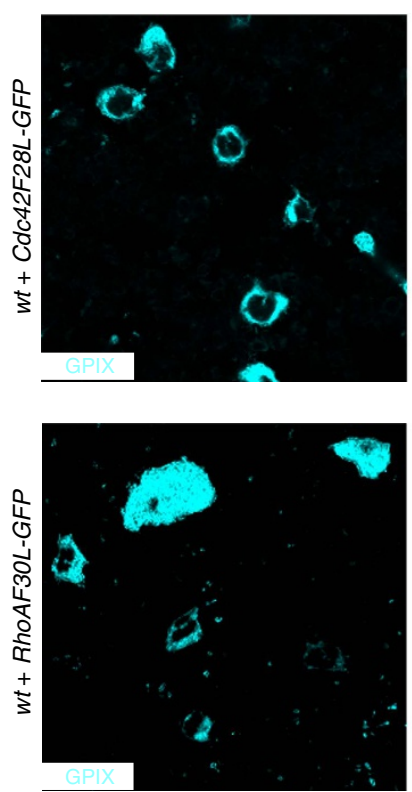
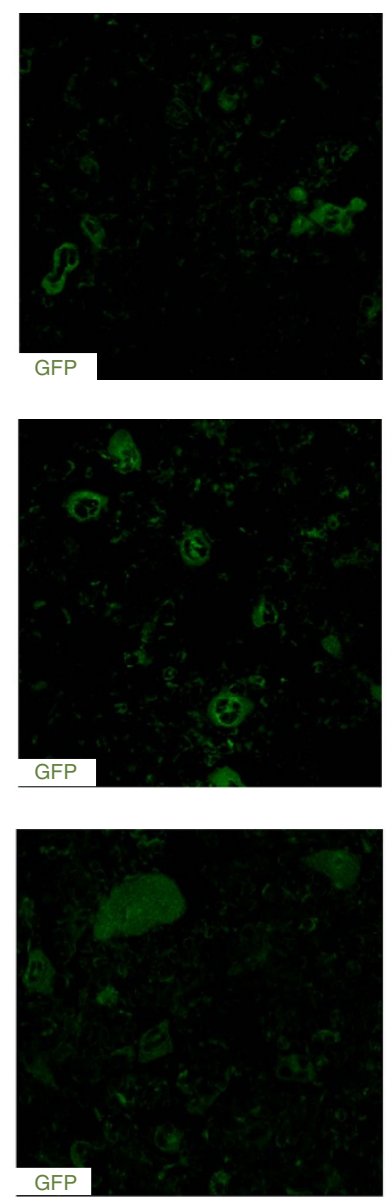

f
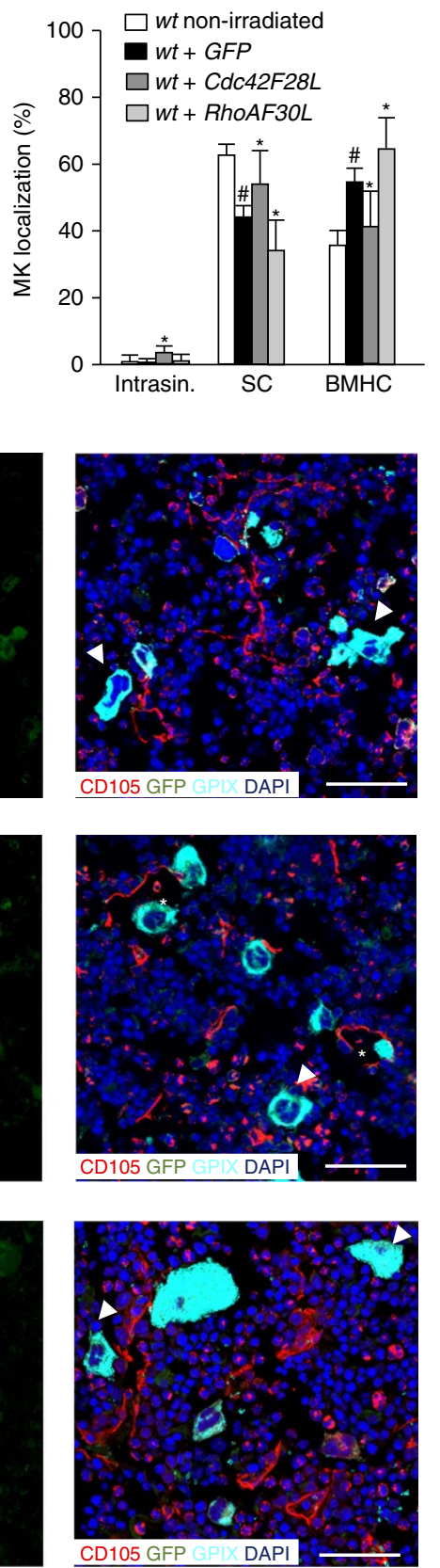

Figure 7 | Activation states of RhoA and Cdc42 have opposing functions in MK localization. (a) Third-generation self-inactivating lentiviral vectors were generated, expressing Cdc42F28L and RhoAF30L, respectively, together with GFP through an IRES, or just GFP under control of the MK-specific human GP6 promoter in the internal position. (b) Work-flow. Lineage marker-negative (Lin -) BM cells were isolated, pre-stimulated for $24 \mathrm{~h}$ and transduced twice. Cells were then transplanted into conditioned recipient mice. (c-e) Representative confocal images of immunostained BM of wt mice after BM transplantation of wt HSC transduced with GFP (c), constitutive active Cdc42 (F28L) (d) or constitutive active RhoA (F30L) (e). Scale bars, $50 \mu$ m. MKs, proplatelets and platelets are shown by GPIX staining in cyan colour. Endoglin staining (red) labels vessels. DAPI, blue. Arrowhead indicates MKs in BMHC, asterisk indicates intrasinusoidal MKs. (f) Quantification of MK localization in the BM $(n=4)$. Quantification of MK localization in non-irradiated wt mice (white) are shown to demonstrate altered localization after irradiation and transplantation of wt cells. Bar graphs represent mean \pm s.d. Two-way ANOVA with Bonferroni correction for multiple comparisons. ${ }^{\star} P<0.05$, compared to $w t+\mathrm{GFP}$; ${ }^{*} P<0.05$, compared to wt not transplanted.

GPIb-IX-V complex and multiple cytoskeletal proteins including Filamin A, non-muscle myosin IIa (NMM-IIa), $\beta$-tubulin, $\alpha$-actinin and Diaphanous Related Formin 1 (DIAPH1) that control the late stages of MK maturation with defects in individual proteins all giving rise to macrothrombocytopenia ${ }^{40,44}$. Interaction of the cytoplasmic domain of GPIb $\alpha$ with Filamin A provides a major link between the receptor and the MK cytoskeleton and coordinated expression of GPIb $\alpha$ and Filamin
A is required for proper localization of either protein ${ }^{45}$. Deficiency of Filamin A in MKs was shown to affect DMS formation $^{46}$ and results in premature release of large, fragile platelets in humans and mice ${ }^{47,48}$. Together with the observation that polarity in osteoclasts depends on a network involving both Filamin A and Cdc42 (ref. 49) this points to a critical role of Filamin A for the transmission of signalling between GPIb $\alpha$ and Cdc42. 
a

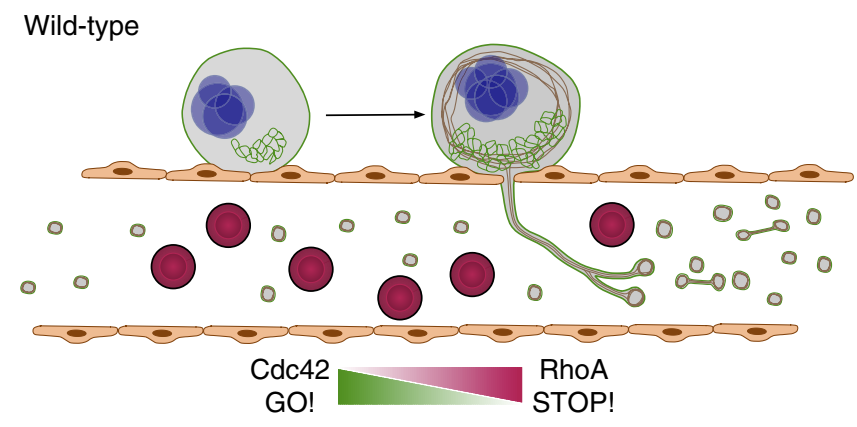

b

GPIb $\alpha / C d c 42$ signalling impaired

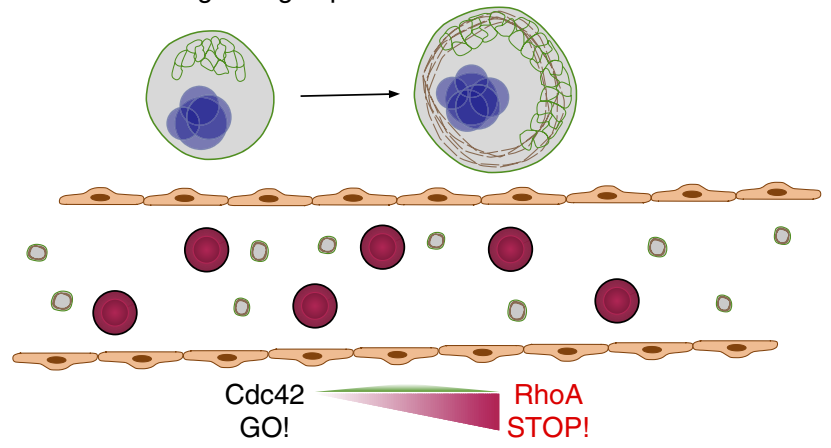

C

RhoA signalling impaired

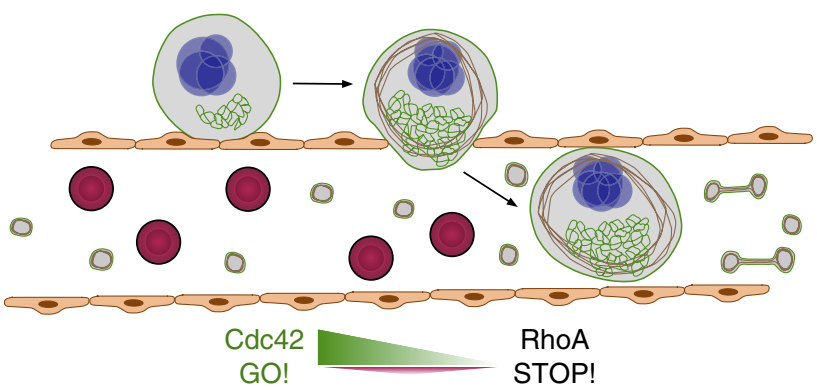

Figure 8 | Model of the Cdc42/RhoA regulatory circuit controlling GPIb $\alpha$-dependent platelet biogenesis. (a) Wt BM MKs polarize their DMS as prerequisite for directed proplatelet release into sinusoidal vessels. This polarization is driven by locally active $\mathrm{Cdc} 42$. (b) Perturbed intracellular GPIb $\alpha$-signalling reduces local $\mathrm{Cdc} 42$ activity and results in defective DMS polarization, reduced MK localization at BM sinusoids and decreased transendothelial proplatelet formation. (c) In the absence of RhoA, Cdc42 activity is locally increased resulting in hyperpolarized MKs entirely transmigrating through the endothelial barrier into sinusoidal vessels.

Our data from RhoA-deficient mice clearly exclude that size restrictions limit $\mathrm{MK}$ transmigration through $\mathrm{BM}$ sinusoids and emphasize RhoA as a critical regulator of proplatelet extension across the endothelial barrier by providing a 'stop' signal for MK polarization and thus transmigration (Fig. 8). In line with this, it has been described that excessive RhoA activation in BM-derived macrophages leads to decreased polarization, motility and cell spreading ${ }^{50}$. Of note, RhoA-dependent activation of Rho kinase (ROCK) and NMM-IIa was shown to negatively regulate proplatelet formation in MKs derived from CD34 + cells in vitro ${ }^{33}$. Mutations in the gene encoding NMM-IIa, MYH9, affect platelet biogenesis in patients with MYH9-related disorders ${ }^{51}$. Interestingly, $\mathrm{MKs}$ from mice with MK-restricted NMM-IIa deficiency $\left(M y h 9^{-/-}\right)$exhibit increased proplatelet formation in vitro ${ }^{52}$ and a significant number of MK nuclei was observed in lungs of $M y \mathrm{~h}^{-/-}$ animals compared to the wild-type ${ }^{53}$. However, given the multiple intrinsic defects present in $M y h 9^{-/-}$MKs, further studies using different experimental approaches will be required to decipher the role of NMM-IIa in RhoA-induced MK polarization and platelet biogenesis.

In summary, the herein described Cdc42/RhoA regulatory circuit downstream of GPIb ensures a tight control of transendothelial platelet biogenesis in the BM and opens new avenues to treat both inherited and acquired forms of thrombocytopenia that are major causes of bleeding in man, and additionally to modulate in vitro production of platelets in the field of transfusion medicine.

\section{Methods}

Mice. All animal experiments, except studies involving BM transplantation, were carried out in the Institute of Experimental Biomedicine (Würzburg), and approved by the district government of Lower Frankonia (Bezirksregierung Unterfranken). BM transplantation experiments were carried out at the Paul-Ehrlich-Institute (Langen) and approved by the local ethics committee of the Lower Saxony State Office (according to $\$ 15$ German animal protection law). Gplba $a^{-/-}$mice (C57Bl/6 background), Gp1ba ${ }^{-/-; T g}$ (Gplba-tg) mice (C57Bl/6 background), where the ectodomain of GPIb $\alpha$ is replaced by that of the human interleukin-4 receptor $\alpha$ (IL-4R $\alpha$ ), and $P r k c i^{-1}-$ mice (C57Bl/6 background) have been described ${ }^{24,54}$. MK/platelet-specific conditional RhoA- and Cdc42-deficient mice (both mixed C57Bl/6 and SV129 background), carrying the Cre-recombinase under the platelet factor 4 (Pf4) promoter ${ }^{55}$, have been described ${ }^{13,14}$. $R h o A^{-/-}$mice were intercrossed with $G p 1 b a^{-/-; T g}$ mice to generate RhoA $A^{-/-} / G p 1 b a^{-/-; T g}$ mice, with $C d c 42^{-/-}$mice to generate RhoA/Cdc42-/mice, and with $\mathrm{Racl}^{-/-}$mice (mixed $\mathrm{C} 57 \mathrm{Bl} / 6$ and SV129 background) to generate $R h o A / R_{a c 1}{ }^{-/-}$mice. $P r k c i^{-/-}$mice were kindly provided by Dr Michael Leitges ${ }^{54}$. $V w f^{-/}$mice (C57Bl/6 background) were kindly provided by Dr Cecile Denis ${ }^{56}$. Mice used in experiments were 8 to 16 weeks old and sex-matched (both sexes used), if not stated otherwise.

Antibodies and reagents. Anaesthetic drugs (medetomidine (Pfizer), midazolam (Roche) and fentanyl (Janssen-Cilag)) were used according to the regulations of the district government of Lower Frankonia (Bezirksregierung Unterfranken). Mice were bled into high-molecular-weight heparin (Ratiopharm). Mice were treated with the selective PI3K inhibitor wortmannin (Sigma-Aldrich; $1 \mu \mathrm{gg}^{-1}$ body weight; days 1 and 3), the microtubule polymerization inhibitor nocodazole (Sigma-Aldrich; $10 \mathrm{\mu g} \mathrm{g}^{-1}$ body weight; days 1-3), or with monovalent Fab fragments of antibodies directed against the major ligand-binding domain of GPIb $\alpha$ (p0p/B-Fab (ref. 25); $100 \mu$ g per mouse, days 1 and 3), integrin $\alpha \operatorname{IIb} \beta 3$ (JON/A (ref. 36); $100 \mu \mathrm{g}$ per mouse, days 1-3) or GPV (DOM1 (ref. 37); $100 \mu \mathrm{g}$ per mouse, days 1-3). Mice were killed and femora dissected and processed on day 5. Antibodies were fluorescently labelled using protein labelling kits (Alexa Fluor 488/546/647, Life Technologies). Anti-CD105 antibody (120402 (MJ7/18), BioLegend) or Tetramethylrhodamine dextran (2 MDa; Thermo Scientific) were used for BM vasculature staining. All other antibodies were produced and modified in our laboratory ${ }^{37}$. Antibodies were incubated for $6-8 \mathrm{~h}$ with immobilized papain or for $24 \mathrm{~h}$ with immobilized pepsin according to the manufacturer's instructions (Pierce Biotechnology, Inc.), and the preparations were then applied to an immobilized protein A column, followed by an immobilized protein G column (Pharmacia) to remove Fc fragments and undigested IgG. Purity of Fab or F(ab)2 fragments was tested by SDS-PAGE. For control experiments, purified rat IgG2a (Serotec) and nonimmune control rat IgG Fab were used.

Immunofluorescence staining on whole femora cryosections. Femora were isolated, fixed with 4\% PFA (AppliChem) and $5 \mathrm{mM}$ sucrose (Sigma-Aldrich), transferred into $10 \%$ sucrose in PBS and dehydrated using a graded sucrose series. Subsequently, the samples were embedded in Cryo-Gel (Leica Biosystems) and shock frozen in liquid nitrogen. Frozen samples were stored at $-80^{\circ} \mathrm{C}$. Seven-micrometer-thick cryosections were generated using the CryoJane tape transfer system (Leica Biosystems) and probed with Alexa488-conjugated antiGPIX antibody, to specifically label platelets and MKs, and Alexa647-conjugated anti-CD105 antibodies (3.33 $\mathrm{mg} \mathrm{ml}^{-1}, 120402$ (MJ7/18), Biolegend) to stain the endothelium. Nuclei were stained using DAPI (4,6-diamidino-2-phenylindole; $1 \mathrm{mg} \mathrm{ml}^{-1}$, D1306, Invitrogen). Samples were visualized with a Leica TCS SP5 confocal microscope (Leica Microsystems).

TEM of bone marrow megakaryocytes. For TEM of MKs, BM was flushed from 12 - to 16-week-old mice using Karnovsky fixative (2\% PFA, $2.5 \%$ glutaraldehyde 
in $0.1 \mathrm{M}$ cacodylate buffer) and incubated overnight at $4{ }^{\circ} \mathrm{C}$. Subsequently, fatty components of the samples were fixed with $2 \%$ osmium tetroxide in $50 \mathrm{mM}$ sodium cacodylate $(\mathrm{pH} 7.2)$, stained with $0.5 \%$ aqueous uranyl acetate, dehydrated with a graded ethanol series and embedded in Epon 812. Ultra-thin sections were stained with $2 \%$ uranyl acetate (in 100\% ethanol) followed by lead citrate. Images were taken on a Zeiss EM900 TEM (Zeiss). ImageJ software (Wayne Rasband, NIH, USA: http://rsb.info.nih.gov/ij) was used to quantify DMS.

Two-photon intravital microscopy of the BM. Mice were anaesthetized by intraperitoneal injection of medetomidine $0.5 \mu \mathrm{g} \mathrm{g}^{-1}$, midazolam $5 \mu \mathrm{g} \mathrm{g}^{-1}$ and fentanyl $0.05 \mu \mathrm{g} \mathrm{g}^{-1}$ body weight. A $1 \mathrm{~cm}$ incision was made along the midline to expose the frontoparietal skull, while carefully avoiding damage to the bone tissue. The mouse was placed on a customized metal stage equipped with a stereotactic holder to immobilize its head. BM vasculature was visualized by injection of tetramethylrhodamine dextran $\left(8 \mu \mathrm{gg}^{-1}\right.$ body weight, $2 \mathrm{MDa}$, Thermo Scientific). Platelets and MKs were antibody stained with anti-GPIX-AlexaFluor 488 (i.v. injection of $0.6 \mu \mathrm{g} \mathrm{g}^{-1}$ body weight). Images were acquired with a fluorescence microscope equipped with a $\times 20$ water objective with a numerical aperture of 0.95 and a TriM Scope II multiphoton system (LaVision BioTec), controlled by ImSpector Pro-V380 software (LaVision BioTec). Emission was detected with HQ535/50-nm and ET605/70-nm filters. A tunable broad-band Ti:Sa laser (Chameleon, Coherent) was used at $760 \mathrm{~nm}$ to capture Alexa Fluor 488 and rhodamine dextran fluorescence. ImageJ software (NIH) was used to generate movies.

Flow cytometry. Diluted blood (1:20) was incubated for $15 \mathrm{~min}$ at room temperature (RT) with fluorophore-conjugated antibodies $\left(2 \mu \mathrm{g} \mathrm{ml}^{-1}\right)$ directed against platelet surface $\mathrm{GPs}^{57}$. Platelet count and size (forward scatter) were assessed using a FACSCalibur (BD Biosciences) flow cytometer.

Immunoblotting. Denatured platelet lysates were separated by SDS-PAGE and blotted onto polyvinylidene difluoride membranes. Membranes were incubated with anti-RhoA antibody $\left(0.5 \mu \mathrm{g} \mathrm{ml}^{-1}\right.$, Cytoskeleton Inc.), anti-Cdc42 antibody $\left(0.5 \mu \mathrm{g} \mathrm{ml}^{-1}\right.$, Cytoskeleton Inc.) or anti-Racl antibodies $\left(0.5 \mu \mathrm{g} \mathrm{ml}^{-1}\right.$, BD Biosciences) antibodies followed by incubation with appropriate horseradish peroxidase-conjugated secondary antibodies $(1 \mathrm{~h}$, room temperature $)$ and enhanced chemiluminescence solution (JM-K820-500, MoBiTec). Images were recorded using a MultiImage II FC Light Cabinet (Alpha Innotech Corporation) device. As loading control, integrin $\beta 3$ (GPIIIa) levels were determined.

Histology. Three-micrometer-thick sections of formalin-fixed paraffin-embedded spleens and lungs were prepared, deparaffinized and stained with haematoxylin and eosin (MHS32 and 318906, Sigma-Aldrich). MKs were stained with HRP-labelled anti-GPIb $\alpha$ antibodies after antigen retrieval and detected with 3-amino-9-ethylcarbazole substrate. MK number, morphology and localization were analyzed with an inverted Leica DMI 4,000 B microscope.

In vitro BM MK isolation and differentiation. BM cells were obtained from femur and tibia by flushing, and lineage depletion (Lin - ) was performed using $\mathrm{CD} 16 / \mathrm{CD} 32+, \mathrm{Gr} 1+, \mathrm{B} 220+$ and CD11b + antibodies (Biolegend). Lin - cells were cultured in $2.6 \%$ nutrient-supplemented StemPro medium with $2 \mathrm{mM}$ L-glutamine, $100 \mathrm{IU} \mathrm{ml}^{-1}$ penicillin, $50 \mathrm{mg} \mathrm{ml}^{-1}$ streptomycin and $20 \mathrm{ng} \mathrm{ml}^{-1}$ murine stem cell factor, $15 \mathrm{IU} \mathrm{ml}^{-1}$ heparin and $50 \mathrm{ng} \mathrm{ml}^{-1}$ of mTPO at $37^{\circ} \mathrm{C}$ under $5 \% \mathrm{CO}_{2}$ for 3 days. Where indicated, $\mathrm{MK}$ cultures were supplemented daily with $50 \mu \mathrm{g} \mathrm{ml}^{-1}$ of $\mathrm{p} 0 \mathrm{p} / \mathrm{B}-\mathrm{Fab}$ fragment. Mature MKs were enriched by a one-step BSA gradient.

Analysis of MK polarization. For immunofluorescence, MKs in suspension were fixed and permeabilized in one step for $30 \mathrm{~min}$ in PBS with 3.7\% formaldehyde and $0.05 \%$ Triton X-100. Samples were saturated in $1 \%$ fatty acid free BSA in PBS. Incubation with antibodies, fluorescent secondary antibodies, AlexaFluor-labelled phalloidin or DAPI was performed for $1 \mathrm{~h}$ at RT. For 3D imaging, cells were kept in $\mu$-slide ibiTreat chambers (Ibidi) in PBS. Confocal images were captured with a LSM780 operated with Zen software using a $\times 63,1.4$ NA Plan Apochromatic objective lens (Carl Zeiss). Profiling of fluorescence intensity was done with ImageJ $(\mathrm{NIH})$. For 3D-analysis, $\mathrm{z}$-stacks were taken and processed with the Imaris 6.4.2 software (Bitplane AG).

FRET-based measurement of Cdc42 activity. The Raichu-Cdc42 encoding sequence was amplified by PCR using Raichu-1054X plasmid as a template and cloned in frame into the pTRIP-IRES lentiviral vector plasmid using BamHI and NheI restriction sites (underlined) ${ }^{32}$. The primers were $5^{\prime}$-CGCGGATCCTTGG CAAGAATTCGGCATGG-3 (forward) and 5'-CTAGCTAGCGGCAGAGGGAAA AAGATCCGTCGAC-3' (reverse). UT711ocl cells were transduced by incubation with lentiviral particles at a multiplicity of infection (MOI) of 1 for 2 days. Transduction efficiency was checked by fluorescence and reached about $100 \%$ of the population. Transduction of primary MKs was performed on Lin - population at an MOI of 5 for 1 day, then $50 \mathrm{ng} \mathrm{ml}^{-1}$ mTPO were added for 3 additional days. FRET efficiency was represented as the colour-coded ratio image of YFP/CFP after background subtraction using MetaMorph (Universal Imaging). Acquisitions were performed with a LSM780 confocal microscope piloted by the Zen software, using a -63, 1.4 NA Plan Apochromatic lens (Carl Zeiss).

Lentiviral gene transfer of Rho GTPase mutants. The cDNAs of Cdc42F28L or RhoAF30L were inserted into lentiviral vectors upstream of an IRES co-expressing eGFP as reporter (lentiviral vector RRL.PPT.SF.eGFP.pre kindly provided by A. Schambach, Hannover Medical School $\left.{ }^{58}\right)$. Expression was controlled by the MK-specific human GP6 promoter ( $h G P 6,-697$ to +29 (ref. 59)). Lentiviral particles were produced by transient transfection in 293T cells. The day before transfection, $5 \times 10^{6} 293 \mathrm{~T}$ cells were seeded in $10 \mathrm{~cm}$ plates in DMEM medium containing $10 \%$ fetal calf serum, $1 \%$ glutamine, $1 \%$ penicillin/streptomycin, $20 \mathrm{mM}$ HEPES. The next day, 293T cells were transfected by calcium-phosphate transfection method with $10 \mu \mathrm{g}$ of vector plasmid, $10 \mu \mathrm{g}$ gag/pol plasmid (pCDA3.GP.4XC plasmid), $1.5 \mu \mathrm{g}$ plasmid encoding the VSV-G envelope (pMD.G plasmid), $5 \mu \mathrm{g}$ Rev encoding plasmid in DMEM medium supplemented with $25 \mu \mathrm{M}$ of chloroquine. Lentiviral supernatants were collected after 36 and $48 \mathrm{~h}$ post transfection, and concentrated by ultra-speed centrifugation at $80,000 \mathrm{~g} \times 2 \mathrm{~h}$. Concentrated viral particles were resuspended in StemSpan medium (Stem Cell Technologies, Köln, Germany) and stored at $-80^{\circ} \mathrm{C}$. Prior to viral transduction, Lin - BM cells were pre-stimulated for $24-48 \mathrm{~h}$ in StemSpan containing $10 \mathrm{ng} \mathrm{ml}^{-1}$ murine stem cell factor, $20 \mathrm{ng} \mathrm{ml}^{-1}$ murine TPO, $10 \mathrm{ng} \mathrm{ml}^{-1}$ recombinant human FGF-1, $20 \mathrm{ng} \mathrm{ml}^{-1}$ murine IGF2, 1\% penicillin/streptomycin, $2 \mathrm{mM}$ glutamine. $5 \times 10^{5} \mathrm{Lin}-$ cells were transduced twice on two following days with lentiviral vectors on Retronectin (Takara-Clontech, $10 \mu \mathrm{g} \mathrm{cm}^{-2}$ ) coated wells (24-well or 12-well plates, dependent on the number of cells for transduction, with a defined MOI)

BM transplantation. 8-12-week-old female $\mathrm{C} 57 \mathrm{Bl} / 6$ mice were preconditioned with $10 \mathrm{~Gy}$ irradiation and transplanted with $5 \times 10^{5}$ cells. Cell infusion was performed via tail vein injection in a final volume of $150 \mu \mathrm{l}$. All mice were kept in the specified pathogen-free animal facilities of the Paul-Ehrlich-Institute, Langen, Germany.

Statistical analysis. When comparing two experimental groups, data distribution was analysed using the Shapiro-Wilk test. Where indicated, statistical significance between two experimental groups was analysed using an unpaired two-tailed Student's $t$-test. Otherwise, data were analysed using two-way analysis of variance (ANOVA) with Bonferroni correction for multiple comparisons (Prism 7; GraphPad Software). $P$-values $<0.05$ were considered as statistically significant. ${ }^{\star} P<0.05 ;{ }^{*} P<0.01 ;{ }^{* *} P<0.001$ or as otherwise stated. Data are presented as mean \pm s.d.

Data availability. All data generated or analysed during this study are included in this published article (and its Supplementary Information files).

\section{References}

1. Machlus, K. R. \& Italiano, Jr J. E. The incredible journey: from megakaryocyte development to platelet formation. J. cell biol. 201, 785-796 (2013).

2. Schulze, H. et al. Characterization of the megakaryocyte demarcation membrane system and its role in thrombopoiesis. Blood 107, 3868-3875 (2006).

3. Behnke, O. \& Forer, A. From megakaryocytes to platelets: platelet morphogenesis takes place in the bloodstream. Eur. j. haematol. Suppl. 61, 3-23 (1998).

4. Junt, T. et al. Dynamic visualization of thrombopoiesis within bone marrow. Science 317, 1767-1770 (2007)

5. Patel, S. R., Hartwig, J. H. \& Italiano, Jr J. E. The biogenesis of platelets from megakaryocyte proplatelets. J. clin. invest. 115, 3348-3354 (2005).

6. Nishimura, S. et al. IL-1alpha induces thrombopoiesis through megakaryocyte rupture in response to acute platelet needs. J. cell biol. 209, 453-466 (2015).

7. Mohle, R., Bautz, F., Denzlinger, C. \& Kanz, L. Transendothelial migration of hematopoietic progenitor cells. Role of chemotactic factors. Ann. NY Acad. Sci. 938, 26-34 discussion 34-25 (2001).

8. Avecilla, S. T. et al. Chemokine-mediated interaction of hematopoietic progenitors with the bone marrow vascular niche is required for thrombopoiesis. Nat. med. 10, 64-71 (2004).

9. Pitchford, S. C., Lodie, T. \& Rankin, S. M. VEGFR1 stimulates a CXCR4-dependent translocation of megakaryocytes to the vascular niche, enhancing platelet production in mice. Blood 120, 2787-2795 (2012).

10. Zhang, L. et al. A novel role of sphingosine 1-phosphate receptor S1pr1 in mouse thrombopoiesis. J. exp. med. 209, 2165-2181 (2012). 
11. Andre, P. et al. Platelets adhere to and translocate on von Willebrand factor presented by endothelium in stimulated veins. Blood 96, 3322-3328 (2000).

12. Vasudevan, S. et al. Modeling and functional analysis of the interaction between von Willebrand factor A1 domain and glycoprotein Ibalpha. J. biol. chem. 275, 12763-12768 (2000).

13. Pleines, I. et al. Multiple alterations of platelet functions dominated by increased secretion in mice lacking Cdc42 in platelets. Blood 115, 3364-3373 (2010).

14. Pleines, I. et al. Megakaryocyte-specific RhoA deficiency causes macrothrombocytopenia and defective platelet activation in hemostasis and thrombosis. Blood 119, 1054-1063 (2012).

15. Bernard, J. \& Soulier, J. P. Sur une nouvelle variété de dystrophie thrombocytairehémorr ragipare congénitale. La semaine des hopitaux: organe fonde par l'Association d'enseignement medical des hopitaux de Paris 24, 3217-3223 (1948)

16. Lopez, J. A., Andrews, R. K., Afshar-Kharghan, V. \& Berndt, M. C. Bernard-Soulier syndrome. Blood 91, 4397-4418 (1998).

17. Nurden, A. T. \& Caen, J. P. Specific roles for platelet surface glycoproteins in platelet function. Nature 255, 720-722 (1975).

18. Nurden, A. T., Dupuis, D., Kunicki, T. J. \& Caen, J. P. Analysis of the glycoprotein and protein composition of Bernard-Soulier platelets by single and two-dimensional sodium dodecyl sulfate-polyacrylamide gel electrophoresis. J. clin. invest. 67, 1431-1440 (1981).

19. Ware, J. Molecular analyses of the platelet glycoprotein Ib-IX-V receptor. Thromb. haemost. 79, 466-478 (1998).

20. Hourdille, P. et al. Studies on the megakaryocytes of a patient with the Bernard-Soulier syndrome. Br. j. haematol. 76, 521-530 (1990).

21. Poujol, C., Ware, J., Nieswandt, B., Nurden, A. T. \& Nurden, P. Absence of GPIbalpha is responsible for aberrant membrane development during megakaryocyte maturation: ultrastructural study using a transgenic model. Exp. hematol. 30, 352-360 (2002).

22. Ware, J., Russell, S. \& Ruggeri, Z. M. Generation and rescue of a murine model of platelet dysfunction: the Bernard-Soulier syndrome. Proc. Natl Acad. Sci. USA 97, 2803-2808 (2000).

23. Strassel, C. et al. Intrinsic impaired proplatelet formation and microtubule coil assembly of megakaryocytes in a mouse model of Bernard-Soulier syndrome. Haematologica 94, 800-810 (2009).

24. Kanaji, T., Russell, S. \& Ware, J. Amelioration of the macrothrombocytopenia associated with the murine Bernard-Soulier syndrome. Blood 100, 2102-2107 (2002).

25. Kleinschnitz, C. et al. Targeting platelets in acute experimental stroke: impact of glycoprotein Ib, VI, and IIb/IIIa blockade on infarct size, functional outcome, and intracranial bleeding. Circulation 115, 2323-2330 (2007).

26. Jackson, S. P. et al. PI 3-kinase p110beta: a new target for antithrombotic therapy. Nat. med. 11, 507-514 (2005).

27. Ozaki, Y., Asazuma, N., Suzuki-Inoue, K. \& Berndt, M. C. Platelet GPIb-IX-V-dependent signaling. J. thromb. haemost. 3, 1745-1751 (2005).

28. Iden, S. \& Collard, J. G. Crosstalk between small GTPases and polarity proteins in cell polarization. Nat. rev. Mol. cell biol. 9, 846-859 (2008).

29. Etienne-Manneville, S. Cdc42-the centre of polarity. J. cell sci. 117, 1291-1300 (2004).

30. Cox, E. A., Sastry, S. K. \& Huttenlocher, A. Integrin-mediated adhesion regulates cell polarity and membrane protrusion through the Rho family of GTPases. Mol. biol. cell 12, 265-277 (2001).

31. Nobes, C. D. \& Hall, A. Rho, rac, and cdc42 GTPases regulate the assembly of multimolecular focal complexes associated with actin stress fibers, lamellipodia, and filopodia. Cell 81, 53-62 (1995).

32. Antkowiak, A. et al. Cdc42-dependent F-actin dynamics drive structuration of the demarcation membrane system in megakaryocytes. J. thromb. haemost. 14, 1268-1284 (2016).

33. Chang, Y. et al. Proplatelet formation is regulated by the Rho/ROCK pathway. Blood 109, 4229-4236 (2007).

34. Humble, J. G., Jayne, W. H. \& Pulvertaft, R. J. Biological interaction between lymphocytes and other cells. Br. j. haematol. 2, 283-294 (1956).

35. Weyrich, A. S. \& Zimmerman, G. A. Platelets in lung biology. Annu. rev. physiol. 75, 569-591 (2013).

36. Nieswandt, B. et al. Long-term antithrombotic protection by in vivo depletion of platelet glycoprotein VI in mice. J. exp. med. 193, 459-469 (2001).

37. Nieswandt, B., Bergmeier, W., Rackebrandt, K., Gessner, J. E. \& Zirngibl, H. Identification of critical antigen-specific mechanisms in the development of immune thrombocytopenic purpura in mice. Blood 96, 2520-2527 (2000).

38. Lin, R., Cerione, R. A. \& Manor, D. Specific contributions of the small GTPases Rho, Rac, and Cdc42 to Dbl transformation. J. biol. chem. 274, 23633-23641 (1999).

39. Latorre-Rey, L. J. et al. Targeting expression to megakaryocytes and platelets by lineage-specific lentiviral vectors. J. thromb. haemost. 15, 341-355 (2017).

40. Nurden, A. T. \& Nurden, P. Congenital platelet disorders and understanding of platelet function. Br. j. haematol. 165, 165-178 (2014).
41. Kaplan, Z. S. et al. Thrombin-dependent intravascular leukocyte trafficking regulated by fibrin and the platelet receptors GPIb and PAR4. Nat. commun. 6 7835 (2015).

42. Kauskot, A. et al. LIM kinase/cofilin dysregulation promotes macrothrombocytopenia in severe von Willebrand disease-type $2 \mathrm{~B}$. JCI insight 1, e88643 (2016).

43. Etienne-Manneville, S. \& Hall, A. Integrin-mediated activation of Cdc42 controls cell polarity in migrating astrocytes through PKCzeta. Cell 106, 489-498 (2001)

44. Stritt, S. et al. A gain-of-function variant in DIAPH1 causes dominant macrothrombocytopenia and hearing loss. Blood 127, 2903-2914 (2016).

45. Kanaji, T., Ware, J., Okamura, T. \& Newman, P. J. GPIbalpha regulates platelet size by controlling the subcellular localization of filamin. Blood 119, 2906-2913 (2012).

46. Begonja, A. J. et al. FlnA binding to PACSIN2 F-BAR domain regulates membrane tubulation in megakaryocytes and platelets. Blood 126, 80-88 (2015).

47. Jurak Begonja, A., Hoffmeister, K. M., Hartwig, J. H. \& Falet, H. FlnA-null megakaryocytes prematurely release large and fragile platelets that circulate poorly. Blood 118, 2285-2295 (2011).

48. Nurden, P. et al. Thrombocytopenia resulting from mutations in filamin A can be expressed as an isolated syndrome. Blood 118, 5928-5937 (2011).

49. Steenblock, C. et al. The Cdc42 guanine nucleotide exchange factor FGD6 coordinates cell polarity and endosomal membrane recycling in osteoclasts. J. biol. chem. 289, 18347-18359 (2014).

50. Pixley, F. J. et al. BCL6 suppresses RhoA activity to alter macrophage morphology and motility. J. cell sci. 118, 1873-1883 (2005).

51. Nurden, A. T. Qualitative disorders of platelets and megakaryocytes. J. thromb haemost. 3, 1773-1782 (2005).

52. Eckly, A. et al. Abnormal megakaryocyte morphology and proplatelet formation in mice with megakaryocyte-restricted MYH9 inactivation. Blood 113, 3182-3189 (2009).

53. Leon, C. et al. Romiplostim administration shows reduced megakaryocyte response-capacity and increased myelofibrosis in a mouse model of MYH9-RD. Blood 119, 3333-3341 (2012).

54. Seidl, S. et al. Phenotypical analysis of atypical PKCs in vivo function display a compensatory system at mouse embryonic day 7.5. PLOS ONE 8, e62756 (2013).

55. Tiedt, R., Schomber, T., Hao-Shen, H. \& Skoda, R. C. Pf4-Cre transgenic mice allow the generation of lineage-restricted gene knockouts for studying megakaryocyte and platelet function in vivo. Blood 109, 1503-1506 (2007).

56. Denis, C. et al. A mouse model of severe von Willebrand disease: defects in hemostasis and thrombosis. Proc. Natl Acad. Sci. USA 95, 9524-9529 (1998).

57. Dutting, S. et al. Growth factor receptor-bound protein 2 contributes to (hem)immunoreceptor tyrosine-based activation motif-mediated signaling in platelets. Circ. res. 114, 444-453 (2014).

58. Schambach, A. et al. Equal potency of gammaretroviral and lentiviral SIN vectors for expression of O6-methylguanine-DNA methyltransferase in hematopoietic cells. Mol. ther. 13, 391-400 (2006).

59. Holmes, M. L., Bartle, N., Eisbacher, M. \& Chong, B. H. Cloning and analysis of the thrombopoietin-induced megakaryocyte-specific glycoprotein VI promoter and its regulation by GATA-1, Fli-1, and Sp1. J. biol. chem. 277, 48333-48341 (2002).

\section{Acknowledgements}

We thank Jonas Müller, Stefanie Hartmann, Sylvia Hengst and Franziska Schenk for excellent technical assistance and Imke Meyer for initial support during multi-photon microscopy. We are also thankful to the microscopy platform of the Bioimaging Centre (Rudolf Virchow Centre) and of the INSERM UMR1048, especially E. Vega, for providing technical infrastructure and support. This work was supported by the Deutsche Forschungsgemeinschaft (NI 556/9-1 to B.N., SFB688 to D.S. and K.G.H., and PL 707/2-1 to I.P.) and the Rudolf Virchow Center. S.S., J.M.M.v.E., D.C. and A.A.B. were supported by a grant of the German Excellence Initiative to the Graduate School of Life Sciences, University of Würzburg. Development of mice in Z.M.R. laboratory was supported by NIH grant HL-117722 and MERU Foundation, Italy.

\section{Author contributions}

S.D. designed research, performed experiments, analysed data and wrote the manuscript M.P., A.A., T.H., K.A., F.G. and N.H. performed experiments and analysed the data. D.S., S.S., D.C., P.N., U.M., I.P., and F.G.-I. performed experiments, analysed data and wrote the manuscript. J.M.M.v.E. and A.A.B. performed experiments. O.A., M.G. and K.G.H. analysed the data. G.K., A.T.N. contributed to the writing of the manuscript. H.S., J.W., C.B. and Z.M.R. provided vital new reagents and contributed to the writing of the manuscript. B.N. conceived the study, designed research, analysed data and wrote the manuscript. 


\section{Additional information}

Supplementary Information accompanies this paper at http://www.nature.com/ naturecommunications

Competing interests: The authors declare no competing financial interests.

Reprints and permission information is available online at http://npg.nature.com/ reprintsandpermissions/

How to cite this article: Dütting, S. et al. A Cdc42/RhoA regulatory circuit downstream of glycoprotein Ib guides transendothelial platelet biogenesis. Nat. Commun. 8, 15838 doi: 10.1038/ncomms15838 (2017).

Publisher's note: Springer Nature remains neutral with regard to jurisdictional claims in published maps and institutional affiliations. (c) (i) Open Access This article is licensed under a Creative Commons Attribution 4.0 International License, which permits use, sharing, adaptation, distribution and reproduction in any medium or format, as long as you give appropriate credit to the original author(s) and the source, provide a link to the Creative Commons license, and indicate if changes were made. The images or other third party material in this article are included in the article's Creative Commons license, unless indicated otherwise in a credit line to the material. If material is not included in the article's Creative Commons license and your intended use is not permitted by statutory regulation or exceeds the permitted use, you will need to obtain permission directly from the copyright holder. To view a copy of this license, visit http://creativecommons.org/ licenses/by/4.0/

(C) The Author(s) 2017 